\title{
A New Method for State of Charge and Capacity Estimation of Lithium-Ion Battery Based on Dual Strong Tracking Adaptive H Infinity Filter
}

\author{
Zheng Liu $\mathbb{D}^{1,2}$ and Xuanju Dang $\mathbb{D}^{1}$ \\ ${ }^{1}$ School of Electronic and Automation, Guilin University of Electronic Technology, Guilin 541004, China \\ ${ }^{2}$ School of Electronic and Automation, Guilin University of Aerospace Technology, Guilin 541004, China \\ Correspondence should be addressed to Xuanju Dang; xjd69@163.com
}

Received 19 April 2018; Revised 27 July 2018; Accepted 26 August 2018; Published 17 September 2018

Academic Editor: Laurent Dewasme

Copyright (C) 2018 Zheng Liu and Xuanju Dang. This is an open access article distributed under the Creative Commons Attribution License, which permits unrestricted use, distribution, and reproduction in any medium, provided the original work is properly cited.

\begin{abstract}
As one of the most important features representing the operating state of power battery in electric vehicles (EVs), state of charge (SOC) and capacity estimation is a crucial assessment index in battery management system (BMS). This paper presents a fusion method of SOC and capacity estimation with identified model parameters. The equivalent circuit model (ECM) parameters are obtained online by variable forgetting factor recursive least squares (VFFRLS), which is based on incremental ECM analysis to respond to the inconsistent rates of parameters variation. The independent open-circuit voltage (OCV) estimation way is designed to reduce the effect of mutual coupling between OCV and ECM parameters. Based on the identified ECM parameters and OCV, a dual adaptive $\mathrm{H}$ infinity filter (AHIF) combined with strong tracking filter (STF) is proposed to estimate battery SOC and capacity. A new quadratic function as capacity error compensation is introduced to represent the relationship between capacity and OCV. The adaptive strategy of the AHIF can adjust noise covariance and restricted factor, while the STF can regulate prior state covariance by adding suboptimum fading factor. The results of experiment and simulation show the merits of proposed approach in SOC and capacity estimation.
\end{abstract}

\section{Introduction}

For the sustainable development strategy of electric vehicles (EVs), the rechargeable lithium-ion battery has been extensively investigated in BMS for EVs in recent years [1, 2]. Reliable BMS is established to ensure the operational safety and enhance the working performance in high energy EVs. The research focuses on lithium-ion battery qualities, especially SOC and instantaneous capacity, both of which are two of the most crucial functions in the BMS, to provide the essential basis for state of health $(\mathrm{SOH})$ and $\mathrm{EV}$ s driving safety $[3,4]$. Since lithium-ion battery can be thought of as a strong nonlinear system with complex electrochemical characteristics, the SOC and capacity cannot directly measured by sensors, while they can be estimated by utilizing ECM-based mathematical method and so on. Therefore, a reliable and high precision joint estimation method of SOC and instantaneous capacity is significant for EVs applications $[5,6]$.

Various methods concentrating on the estimation of SOC have been developed over the past few years. The traditional classical approach is open-circuit voltage (OCV) measurement $[7,8]$ using fitting function of OCV-SOC in the charge and discharge experiments. Because the battery's steady state cannot be achieved until the long rest time, OCV method is not suitable for real-time SOC estimation. Another approach is the Ampere-Hour counting $(\mathrm{AH})$ method $[9,10]$ with speed calculation and simple implementation. However, an accurate initialization SOC and the high precision measurement current are required simultaneously. As an open loop method, AH with accumulative error and rounding error is unavoidable in practical measurement, such as diffusion and drift current. In addition, the black box-based forecasting technique, such as the artificial neural network (ANN) [11, 
12], the fuzzy logic [13], and the support vector machine (SVM) $[14,15]$, does not demand accurate measurement data, but is limited by many factors like heavy training burden and being very time consuming. Compared with electrochemical model based method, ECM-based simplified approach has the advantages of low computational complexity and high estimation precision. As one reduced form of complex electrochemical model, the ECM has been extensively researched to describe dynamic hysteresis characteristics of lithiumion battery through composition of basic electrical circuit elements. In [16, 17], the dynamic response of lithium-ion battery can be simulated by one-order RC ECM with a simple topology structure, and a recursive least squares (RLS) method is presented for parameters identification. However, the RLS with constant forgetting factor mismatches the ECM parameters with different changing rates.

In the ECM-based state observer method, the flexible usage of Kalman filter (KF) which assumes some special properties of model error-free and known noise statistics has been generally accepted for its better performance of optimal estimation. For example, the extended Kalman filter (EKF) is applied for lithium-ion battery parameters identification [18]. However, the error of first order Taylor series approximation results in slowness of the convergence rate or even in filtering divergence. The unscented Kalman filter (UKF) is utilized to estimate lithium-ion battery SOC $[18,19]$, and the estimation results show that the UKF has better robustness and higher precision than the EKF. The adaptive EKF (AEKF) and adaptive UKF (AUKF) are adopted in state estimator to achieve the goal of higher precision and better stability than EKF and UKF $[20,21]$. The $\mathrm{H}$ infinity filter (HIF) is also applied to the model parameters identification and state estimation [2227] and has better performance than UKF and EKF. Even so, for the above filtering method based on ECM with model uncertainty, the obvious disadvantages can be represented as oversimplified ECM, unknown noise characteristics, and fast (slow) time-varying model parameters [28-30], which can show that ECM cannot fully reflect battery dynamic behavior. Therefore, the tracking performance of ECM-based filtering approach becomes poor or even there exists filtering divergence. To overcome this weakness, the STF and KFs are combined to estimate battery state. In [31], the cubature Kalman filter (CKF) with STF is introduced to improve the robustness and accuracy of SOC estimation. A strong tracking estimator is proposed in [32], and the model parameters are identified with genetic algorithm; meanwhile a strong tracking UKF is used to estimate SOC. A composite filtering method is proposed in [20], the criterion of selecting proper innovation flows following chi-square distribution has been introduced to define model uncertainty, and a combination algorithm of strong tracking UKF and adaptive UKF has been developed to estimate SOC. In order to ensure that the covariance matrix always keeps nonnegative definiteness matrix, square root CKF plus STF is proposed to estimate SOC in [33], which can restrain the divergence effectively.

In addition to SOC, the capacity representing the age of battery is also an essential part in BMS. Only when capacity is assumed to be known and the value remains the same, the ECM-based SOC estimation method can be used to achieve good performance. However, it is inevitable for the capacity to fade with battery aging. In order to get a more accurate SOC estimation, the capacity has to be updated exactly. To address this problem, some ways have been investigated to estimate real-time capacity [34]. The difference between $\mathrm{SOC}_{1}$ and $\mathrm{SOC}_{2}$ is utilized to calculate capacity online through using OCV-SOC fitting function [35]. The calculated results depend on high precision current which is difficult to be obtained in actual working conditions. In [36], the capacity fitting models are time consuming since they get capacity estimation by a set of cycle experimental data. In general, the capacity is either as an expanding model parameter or as an additional state to be estimated with various kinds of observers. Dual EKF has been used to estimate SOC and capacity simultaneously with certain model parameters in [37]. The obvious drawback of this approach is that the offline parameters cannot represent all the dynamic aspects of operating condition and aging situation. In [38-40], the capacity is integrated into a set of parameters, and then the SOC and capacity are estimated with dual EKF. The experimental results show its robustness to operating condition and aging situation with assistance of adaptive updating parameters. Some types of proportionalintegral observers are used to estimate parameters, SOC, and capacity simultaneously based on electrochemical model [41]. A combined estimator based on EKF is introduced in [42], the model parameters are identified by adaptive RLS, and then SOC and capacity are estimated with reduced order EKF. In [43] a single EKF is used to estimate SOC, and RLS is designed to estimate capacity. The dimension of matrix is decreased, relative to combined estimator. Significantly, the model uncertainty which causes negative effect on estimation performance has not been addressed through the abovementioned methods.

Compared with the existing researches, the main contributions of this paper include the following: (1) a simplified ECM is established, and the VFFRLS method based on incremental model analysis is designed to identify the battery model parameters online; (2) an independent OCV estimator excluding model parameters is adopted to accurately capture the OCV; (3) with the adaptive HIF (AHIF) including noise covariance and restricted factor adjustment, the SOC and capacity are directly estimated; and (4) a new dual strong tracking AHIF (ST-AHIF) method with capacity error compensation (EC) for SOC and capacity estimation is introduced to improve the robustness and precision of the AHIF algorithm. Compared with the wide usage of AEKF and AHIF, the proposed method has the features of strong robustness and high precision.

The rest of this paper is arranged as follows. Section 2 presents details of derivation of battery ECM and the model parameters identification based on VFFRLS with incremental model analysis. The independent OCV estimator without model parameters is presented in Section 3. Then based on the identified parameters, a dual ST-AHIF based SOC and capacity joint estimator including EC is proposed in Section 4. Section 5 compares the experimental and simulation results under the DST and FUDS conditions. Finally, some conclusions are drawn in Section 6. 


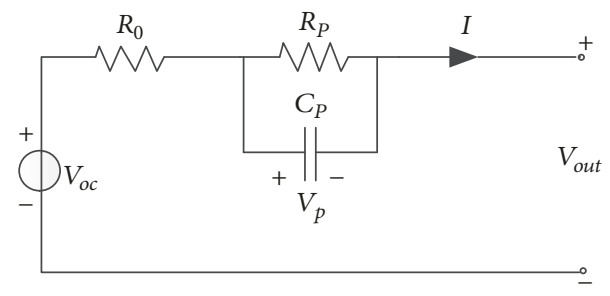

FIgURE 1: Schematic diagram of Thevenin ECM.

\section{Battery Model and Parameters Identification}

The simplified battery model and corresponding parameters identification based on incremental analysis are detailed in this section.

2.1. Battery Model Derivation. Considering the trade-off between model complexity and estimated accuracy, the dynamic electrical characteristic of the lithium-ion battery can be represented by the one-order Thevenin ECM [18], as shown in Figure 1.

According to circuit principle, Thevenin ECM can be expressed as

$$
\begin{gathered}
\dot{V}_{p, t}=-\frac{V_{p, t}}{R_{p} C_{p}}+\frac{I_{t}}{C_{p}} \\
V_{\text {out }, t}=V_{o c, t}-V_{p, t}-R_{0} I_{t}
\end{gathered}
$$

where $V_{o c}$ is used to describe OCV associated with SOC, $R_{0}$ indicates the ohmic resistance, the parallel $R_{P} C_{p}$ composed of a polarization resistance $R_{P}$ and a polarization capacitance $C_{p}, V_{p}$ represents polarization voltage across $R_{p} C_{p}$, I is the charging-discharging current, and $V_{\text {out }}$ represents the terminal voltage.

The nonlinear time series forecasting method is adopted to analyze ECM. Let $\mathrm{u}$ and $\mathrm{y}$ be the charge-discharge current and the terminal voltage of ECM, respectively. The ECM in (1) to be identified can be redefined as a multivariable regression expression:

$$
\begin{aligned}
\mathrm{y}_{k}= & \mathrm{a}_{1} \mathrm{y}_{k-1}+\mathrm{a}_{2} \mathrm{y}_{k-2}+\cdots+\mathrm{a}_{p} \mathrm{y}_{\mathrm{k}-\mathrm{p}+1}+\mathrm{b}_{1} \mathrm{u}_{\mathrm{k}-1} \\
& +\mathrm{b}_{2} \mathrm{u}_{\mathrm{k}-2}+\cdots+\mathrm{b}_{q} \mathrm{u}_{\mathrm{k}-\mathrm{q}+1}
\end{aligned}
$$

where $\mathrm{p}$ and $\mathrm{q}$ are the orders of regression expression and $a_{1}, a_{2}, \ldots a_{p}$ and $b_{1}, b_{2}, \ldots b_{q}$ are the undetermined coefficient.

2.2. Parameters Identification. By comparing (1) with (2), the polarization voltage $V_{p}$ can be eliminated from (1). The discretization form of (1) by bilinear transformation method: $s=\left(2 / T_{s}\right)\left(\left(1-z^{-1}\right) /\left(1+z^{-1}\right)\right)(\mathrm{z}$ is the discretization operator $)$ is given as

$$
V_{\text {out }, k}=a_{1} V_{\text {out }, k-1}+b_{1} I_{k}+b_{2} I_{k-1}+\left[V_{o c, k}-a_{1} V_{o c, k-1}\right]
$$

where $V_{o c, k}, V_{o u t, k}$, and $I_{k}$ indicate the OCV, terminal voltage, and load current at the kth sampling time, respectively, and the corresponding coefficient can be obtained by $a_{1}=$ $\left(2 R_{p} C_{p}-1\right) /\left(1+2 R_{p} C_{p}\right), b_{1}=-\left(2 R_{0} R_{p} C_{p}+R_{0}+R_{p}\right) /(1+$ $\left.2 R_{p} C_{p}\right), b_{2}=\left(2 R_{0} R_{p} C_{p}-R_{0}-R_{p}\right) /\left(1+2 R_{p} C_{p}\right)$. Then $R_{0}, R_{p}$, and $C_{p}$ can be obtained according to the inverse equations of $a_{1}, b_{1}$, and $b_{2}$; thus, $R_{0}=\left(b_{2}-b_{1}\right) /\left(1+a_{1}\right)$, $R_{\mathrm{p}}=2\left(b_{2}+a_{1} b_{1}\right) /\left(a_{1}^{2}-1\right), C_{p}=-\left(a_{1}+1\right)^{2} / 4\left(b_{2}+a_{1} b_{1}\right)$.

By comparing (3) with (2), the immeasurable part $\left(V_{o c, k}-\right.$ $\left.a_{1} V_{o c, k-1}\right)$ is assumed as the residual model error (RME) of ECM.

Similarly, the terminal voltage of the previous step (k-1) could be expressed as

$$
\begin{aligned}
V_{\text {out }, k-1}= & a_{1} V_{\text {out }, k-2}+b_{1} I_{k-1}+b_{2} I_{k-2} \\
& +\left(V_{o c, k-1}-a_{1} V_{o c, k-2}\right) .
\end{aligned}
$$

By subtracting (3) from (4), the incremental equation will be derived as

$$
\begin{aligned}
\Delta V_{\text {out }, k}= & a_{1} \Delta V_{\text {out }, k-1}+b_{1} \Delta I_{k}+b_{2} \Delta I_{k-1} \\
& +\Delta\left(V_{o c, k}-a_{1} V_{o c, k-1}\right)
\end{aligned}
$$

where $\Delta V_{\text {out }, k}, \Delta I_{k}$, and $\Delta\left(V_{o c, k}-a_{1} V_{o c, k-1}\right)$ are calculated as $\left(V_{o u t, k}-V_{o u t, k-1}\right),\left(I_{k}-I_{k-1}\right)$, and $\left(V_{o c, k}-a_{1} V_{o c, k-1}\right)-\left(V_{o c, k-1}-\right.$ $\left.a_{1} V_{o c, k-2}\right)$, respectively. Meanwhile the immeasurable part $\Delta\left(V_{o c, k}-a_{1} V_{o c, k-1}\right)$ is also assumed as the RME of ECM based on incremental analysis.

From (5), it is seen that the $\mathrm{y}$ and $\mathrm{u}$ of the ECM are $\Delta V_{\text {out }, k}$ and $\Delta I_{k}$, respectively. In accordance with nonlinear regression mode principle, (5) can be rewritten as least squares form:

$$
\Delta V_{o u t, k}=\phi_{k}^{T} \theta_{k}
$$

where

$$
\begin{aligned}
\phi_{k} & =\left[\begin{array}{llll}
\Delta V_{\text {out }, k} & \Delta I_{k} & \Delta I_{k-1} & 1
\end{array}\right] \\
\theta_{k} & \left.=\left[\begin{array}{llll}
a_{1} & b_{1} & b_{2} & \Delta\left[V_{o c, k}-a_{1} V_{o c, k}\right.
\end{array}\right]\right]^{T} .
\end{aligned}
$$

In (6), $\theta_{k}$ is the unknown parameter and $\phi_{k}$ is the known coefficient determined by measurement.

The RLS is often used to solve the regression model described in (6). However, the RLS with constant forgetting factor $\lambda$ may encounter the difficulties of balancing between stability and convergence. Seeking to address this problem, we apply the VFFRLS with variable forgetting factors [4446] for identification in this paper. The process of parameters estimation of VFFRLS is realized as follows:

$$
\begin{aligned}
e_{k} & =y_{k}-\phi_{k}^{T} \hat{\theta}_{k-1} \\
\lambda_{\mathrm{k}} & =1-\frac{1}{1+\phi_{k-1-n}^{T} P_{k-1} \phi_{k-1-n}} \frac{e_{k}^{2}}{\sigma^{2}} \\
K_{\mathrm{k}} & =\frac{P_{k-1} \phi_{k}^{T}}{\lambda+\phi_{k}^{T} P_{k-1} \phi_{k}}
\end{aligned}
$$




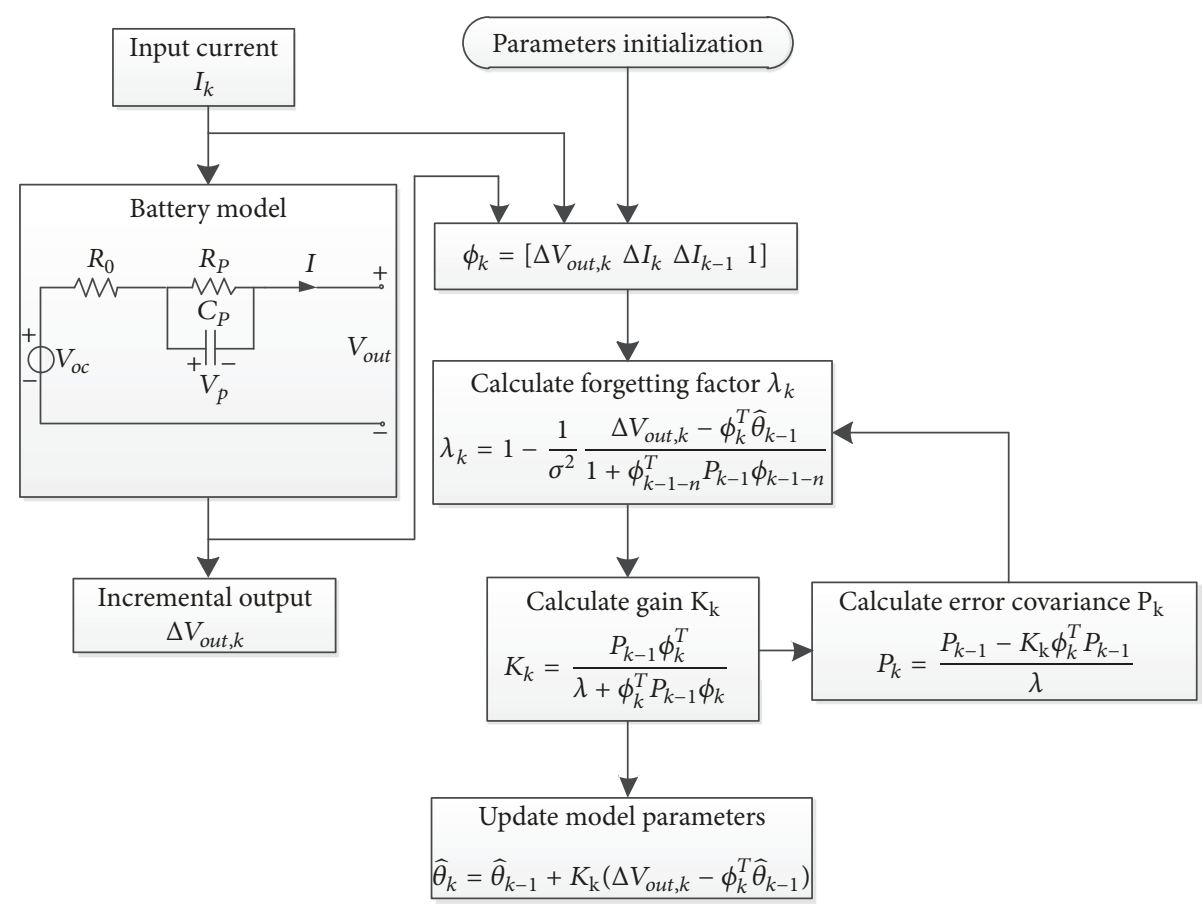

FIgURE 2: Parameters identification algorithm for the Thevenin ECM.

$$
\begin{aligned}
& P_{k}=\frac{P_{k-1}-K_{\mathrm{k}} \phi_{k}^{T} P_{k-1}}{\lambda} \\
& \widehat{\theta}_{k}=\widehat{\theta}_{k-1}+K_{\mathrm{k}} e_{k}
\end{aligned}
$$

where $\widehat{\theta}_{k}$ is the identification of the parameters matrix, $e_{k}$ is the prediction error of the terminal voltage, $K_{\mathrm{k}}$ is the gain vector, $P_{k}$ is the covariance matrix, $\lambda_{\mathrm{k}}$ is the variable forgetting factor, $n$ is the forgetting step, $\sigma^{2}$ is the expected measurement variance, and $P_{0}=10^{-4} I$.

With the VFFRLS, the forgetting factors can be decoupled and tuned separately to improve the parameters stability and tracking accuracy of SOC estimation. The procedure of identification method is illustrated in Figure 2.

\section{Independent OCV Estimator}

In this section, an independent OCV estimator is introduced. The OCV is observed with the aid of battery ECM characterization. The $V_{p, t}$ in (1) can be rewritten in the discrete-time form.

$$
V_{p, k}=e^{-1 / R_{p} C_{p}} V_{p, k-1}+\left(1-e^{-1 / R_{p} C_{p}}\right) R_{p} I_{k-1}
$$

From (1), obviously $V_{p, k}$ can be expressed in the following discrete-time relationship.

$$
V_{p, k}=V_{o c, k-1}-V_{o u t, k-1}-R_{0} I_{k-1}
$$

Substituting (10) into (9) yields the following.

$$
\begin{aligned}
V_{o c, k}= & e^{-1 / R_{p} C_{p}} V_{o c, k-1}-e^{-1 / R_{p} C_{p}}\left(V_{o u t, k-1}+R_{0} I_{k-1}\right) \\
& +\left(1-e^{-1 / R_{p} C_{p}}\right) R_{p} I_{k-1}+V_{o u t, k}+R_{0} I_{k}
\end{aligned}
$$

Since the OCV can be described as a slow time-varying variable, the OCV can be solved by the expression of $R_{0}, R_{p}$, and $C_{p}$ as follows.

$$
\widehat{V}_{\text {oc }, k}=\frac{V_{\text {out }, k}+R_{0} I_{k}-e^{-1 / R_{p} C_{p}}\left(V_{o u t, k-1}+R_{0} I_{k-1}\right)+\left(1-e^{-1 / R_{p} C_{p}}\right) R_{p} I_{k-1}}{1-e^{-1 / R_{p} C_{p}}}
$$

Once the OCV is obtained, the look-up table of OCV-SOC can be easily obtained under high precision experimental conditions [42]. The nonlinear characteristic relationship of OCV-SOC is built in processing the experimental data by the polynomial curve fitting methods.

$$
V_{o c}(k)=\sum_{i=0}^{N} d_{i} \operatorname{SOC}(k)^{i}
$$


TABLE 1: Summary of HIF with tunable restricted factor.

\begin{tabular}{lr}
\hline (1) Initialization & $x_{0}, \gamma_{0}, P_{0}, S_{0}, Q_{0}, R_{0}$ \\
\hline (2) State estimation & $\widehat{x}_{k \mid k-1}=F_{k \mid k-1} \widehat{x}_{k-1 \mid k-1}+B_{k-1} I_{k-1}$ \\
\hline Prior state estimation & $P_{k \mid k-1}=F_{k \mid k-1} P_{k-1 \mid k-1} F_{k \mid k-1}^{T}+Q_{k-1}$ \\
\hline Prior state covariance & $\bar{S}_{k}=L_{k}^{T} S_{k} L_{k}$ \\
\hline Symmetric positive definite matrix update & \\
\hline (3) Measurement correction & $K_{k}=P_{k \mid k-1}\left(I-\gamma_{k} S_{k} P_{k \mid k-1}+H_{k}^{T} R_{k}^{-1} H_{k} P_{k \mid k-1}\right)^{-1} H_{k}^{T} R_{k}^{-1}$ \\
\hline Gain matrix update & $\widehat{x}_{k \mid k}=\widehat{x}_{k \mid k-1}+K_{k}\left(y_{k}-F_{k} \widehat{x}_{k \mid k-1}-D_{k} I_{k}\right)$ \\
\hline Posterior state update & $P_{k \mid k}=P_{k \mid k-1}\left(I-\gamma \bar{S}_{k} P_{k \mid k-1}+H_{k}^{T} R_{K}^{-1} H_{k} P_{k \mid k-1}\right)^{-1}+R_{k}$ \\
\hline Posterior state covariance update & $\gamma_{k}=\alpha_{1} \sqrt{\lambda_{\max }\left(L_{k}^{T} L_{k}\left(P_{k \mid k}^{-1}+F_{k}^{T} F_{k}\right)^{-1}\right)}$ \\
\hline Tunable restricted factor update & $\alpha_{1}=1+\frac{\beta_{1}}{\sqrt{e_{k}^{T} e_{k}}}$ \\
\hline
\end{tabular}

where $\mathrm{V}_{\mathrm{oc}}$ is the OCV, $d_{i}$ is the polynomial fitting coefficient, $\mathrm{N}$ is the order of fitting function, and $\mathrm{N}$ is set to 8 .

\section{Dual ST-AHIF Based SOC and Capacity Integrated Estimator}

4.1. Tunable Restricted Factor Derivation. In general, the deterministic model and known noise statistics is the precondition to bring into play the KFs advantages. However, the KFs cannot be guaranteed to work steadily all the time when these conditions cannot be satisfied. The $\mathrm{H}$ infinity filter (HIF), which is designed to minimize the maximum estimation error, can make up those inadequate aspects; thus it is commonly used for identifying system parameters [22]. The cost function of HIF is defined as shown below:

$$
\begin{aligned}
& J_{1} \\
& =\frac{\sum_{k=0}^{N-1}\left(x_{k}-\widehat{x}_{k}\right) S_{k}\left(x_{k}-\widehat{x}_{k}\right)^{T}}{\left(x_{0}-\widehat{x}_{0}\right) P_{0}^{-1}\left(x_{0}-\widehat{x}_{0}\right)^{T}+\sum_{k=0}^{N-1}\left(w_{k} Q_{k}^{-1} w_{k}^{T}+v_{k} R_{k}^{-1} v_{k}^{T}\right)}
\end{aligned}
$$

where $x_{k}$ is the state value, $\widehat{x}_{k}$ is state estimation, and $x_{0}$ and $\hat{x}_{0}$ are the initial states values. $w_{k}$ and $v_{k}$ are the process noise vector and measurement noise vector, respectively. $P_{0}, Q_{k}, R_{k}$, and $S_{k}$ are the symmetric positive definite matrixes.

Since it is difficult to directly minimize cost function, a user-specified restricted factor $\gamma^{2}$ which is set to a fixed value is preset to guarantee an optimized boundary constrained condition [24], making sure that $J_{1}$ satisfies $J_{1}<\gamma^{2}$. For ensuring both robustness and precision, the self-adapting restricted factor is incorporated into HIF.

By applying the matrix inversion lemma to $P_{k \mid k}, P_{k \mid k}$ should be positive definiteness.

$$
P_{k \mid k}^{-1}=L_{k}^{T} L_{k}\left(P_{k \mid k-1}^{-1}+F_{k}^{T} F_{k}\right)^{-1}-\gamma^{-2} I \geq 0
$$

In other words, the restricted factor $\gamma$ should satisfy

$$
\gamma^{2} \geq \lambda_{\max }\left(L_{k}^{T} L_{k}\left(P_{k \mid k-1}^{-1}+F_{k}^{T} F_{k}\right)^{-1}\right)
$$

where $\lambda_{\max }(A)^{-1}$ denotes the greatest eigenvalue of matrix $(A)^{-1}$. Therefore, self-adapting method for restricted factor $\gamma$ is

$$
\gamma=\alpha_{1} \sqrt{\lambda_{\max }\left(L_{k}^{T} L_{k}\left(P_{k \mid k-1}^{-1}+F_{k}^{T} F_{k}\right)^{-1}\right)}
$$

where $\alpha_{1}$ is used to correct $\gamma$ and $\alpha_{1}>1$; the quadratic sum of innovation error $e_{k}$ is used as estimation error $e_{k}^{T} e_{k}$. As the restricted factor $\gamma$ is inversely proportional to the estimation error, the correction coefficient expression is shown as follows:

$$
\alpha_{1}=1+\frac{\beta_{1}}{\sqrt{e_{k}^{T} e_{k}}}
$$

where $\beta_{1}$ is an unknown coefficient associated with experiments and $\beta_{1}>0$; once the $\beta_{1}$ is determined, the correction coefficient $\alpha_{1}$ is only dependent on innovation error $e_{k}$.

The calculation process of the HIF with tunable restricted factor is shown in Table 1.

4.2. Adjustable Noise Covariance Derivation. Multiple adaptive methods have been integrated into original KFs such as the correlation method, maximum likelihood criterion, and covariance matching method. As mentioned before, the HIF approaches KF when $\gamma \longrightarrow \infty$. Hence it can be concluded that the noise covariance matrix $Q_{k}$ and $R_{k}$ in HIF should be adjusted properly. Therefore, an adaptive HIF with the maximum likelihood criterion is designed to update the noise covariance at each stage of measurement correction.

The innovation error can be written as an expression of difference between measurement estimation and true measurement value.

$$
\mathrm{e}_{k}=y_{k}-\left(H_{k} \widehat{x}_{k \mid k-1}+D_{k} I_{k}\right)=H_{k}\left(x_{k}-\widehat{x}_{k \mid k-1}\right)+v_{k}
$$

The measurement innovation covariance is deduced through probability theory.

$$
\mathrm{P}_{\mathrm{e}_{k}}=C_{k} P_{k \mid k-1} C_{k}^{T}+\widehat{R}_{k}
$$


The definition of innovation estimation variance $C_{k}$ is introduced to describe $\mathrm{P}_{\mathrm{e}_{k}}$ through using moving window method of innovation.

$$
C_{k}=\frac{1}{L} \sum_{j=k-L+1}^{k} \mathrm{e}_{j} \mathrm{e}_{j}^{T}
$$

By combining (20)-(21), the following observation noise covariance equation can be drawn.

$$
\widehat{R}_{k}=C_{k}+H_{k} P_{k \mid k-1} H_{k}^{T}
$$

Similarly, the state noise can be expressed as follows.

$$
w_{k-1}=x_{k}-F_{k-1} x_{k-1}
$$

Substituting state a priori estimate $\widehat{x}_{k \mid k-1}$ into (23) yields the following.

$$
w_{k-1}=\left(x_{k}-\widehat{x}_{k \mid k}\right)-F_{k-1}\left(x_{k-1}-\widehat{x}_{k-1 \mid k-1}\right)+K_{k} e_{k}
$$

Based on the principle of orthogonality between the innovation and the residual, the state noise covariance $\widehat{Q}_{k}$ is taken on both sides of (24).

$$
\widehat{Q}_{k}=F_{k-1} P_{k \mid k-1} F_{k-1}^{T}+K_{k} C_{k} K_{k}^{T}
$$

The iteration approach detailed in (22) and (25) is utilized to compensate for process and measurement noises uncertainties in HIF algorithm.

4.3. STF Derivation. Although HIF has advantages of good robustness to model uncertainty, it loses fast tracking capability for state with abrupt fluctuation when the estimator reaches a stable state. More specifically, when the estimator suffers abnormal disturbance, the gain matrix of HIF will not increase rapidly with the growing of residual error but will still be close to a minimum. To solve this problem, strong tracking filter (STF) is introduced in HIF to regulate gain matrix with incorporating fading factor into prior state covariance matrix.

The basic idea of STF based on orthogonality principle is to select an appropriate filter gain online and satisfy the following requirements:

$$
\begin{aligned}
E\left[\left(x_{k}-\widehat{x}_{k}\right)\left(x_{k}-\widehat{x}_{k}\right)^{T}\right] & =\min \\
V_{k} & =E\left[\varepsilon_{k+j} \varepsilon_{k}^{T}\right]=0
\end{aligned}
$$

where $E\left[\left(x_{k}-\widehat{x}_{k}\right)\left(x_{k}-\widehat{x}_{k}\right)^{T}\right]$ represents the minimum state error covariance and $V_{k}$ denotes the residual covariance matrix that keeps mutually orthogonal at arbitrary times.

The residual covariance matrix $V_{k}$ can be defined as

$$
V_{k}= \begin{cases}\varepsilon_{k} \varepsilon_{k}^{T} & k=1 \\ \frac{\rho V_{k-1}+\varepsilon_{k} \varepsilon_{k}^{T}}{1+\rho} & k \geq 2\end{cases}
$$

where output residual $\varepsilon_{k}$ is defined as $\varepsilon_{k}=y_{k}-\widehat{y}_{k \mid k-1}$ and $\rho$ is forgetting factor whose the range of value is from 0 to $1,0.95$ is commonly adopted.

The matrixes $N$ and $M$ are defined in (28), which is used for calculating the fading factor.

$$
\begin{aligned}
N_{k} & =V_{k}-H_{k} Q_{k-1} H_{k}^{T}-\beta R_{k} \\
M_{k} & =H_{k} F_{k-1} P_{k \mid \mathrm{k}-1} F_{k-1}^{T} H_{k}^{T} \\
\mu_{k} & =\max \left(1, \frac{\operatorname{tr}\left[N_{k}\right]}{\operatorname{tr}\left[M_{k}\right]}\right)
\end{aligned}
$$

where $H_{k}$ and $F_{k-1}$ are the measurement and state matrix, respectively, and $Q_{k-1}$ and $R_{k}$ are the noise covariance of state and measurement, respectively. $\mu_{k}$ is called the fading factor which adjusts gain matrix to realize orthogonality principle. $\operatorname{tr}\left[N_{k}\right]$ and $\operatorname{tr}\left[M_{k}\right]$ are the trace of the matrixes $N_{k}$ and $M_{k}$, respectively, which are used for calculating the fading factor $\mu_{k}$.

The new prior state covariance matrix $P_{k \mid k-1}^{*}$ is obtained by introducing fading factor $\mu_{k}$ into original prior state covariance matrix $P_{k \mid k-1}$.

$$
P_{k \mid k-1}^{*}=\mu_{k} F_{k-1} P_{k-1 \mid k-1} F_{k-1}^{T}+Q_{k-1}
$$

Based on the above analysis, the estimator can maintain its ability to track state with abrupt fluctuation or model uncertainty by the combined algorithm of STF with HIF.

4.4. SOC and Capacity Joint Estimator Based on Dual STAHIF. According to ECM in Figure 1, the discretization of (1) and definition formula of SOC can be rewritten as follows:

$$
\begin{aligned}
V_{o u t, k}= & V_{o c, k}-V_{p, k}-R_{0, k} i_{k} \\
V_{p, k}= & e^{-1 / R_{p, k-1} C_{p, k-1}} V_{p, k-1} \\
& +\left(1-e^{-1 / R_{p, k-1} C_{p, k-1}}\right) R_{p, k-1} i_{k-1} \\
\operatorname{SOC}_{k}= & S O C_{k-1}-\frac{\eta T i_{k-1}}{C_{c a p, k}}
\end{aligned}
$$

where $V_{o u t, k}, V_{o c, k}$, and $V_{p, k}$ are the OCV, terminal voltage, and polarization voltage at the sample time $\mathrm{k}$, respectively, and the $C_{c a p, k}$ is the capacity which is considered as an independence state.

From the discrete ECM expression shown in (31), the two state-space equations including SOC and capacity can be described in (32) and (33), respectively.

$$
\begin{aligned}
x_{k} & =f\left(x_{k-1}, \varphi_{k-1}, u_{k-1}\right)+w_{k-1}^{x} \\
& =F_{k-1} x_{k-1}+B_{k-1} i_{k-1}+w_{k-1}^{x} \\
y_{k} & =h\left(x_{k}, \varphi_{k}, u_{k}\right)+v_{k}=H_{k}^{x} x_{k}+D_{k} i_{k}+v_{k}
\end{aligned}
$$




$$
\begin{aligned}
& \varphi_{k}=\varphi_{k-1}+w_{k-1}^{\varphi} \\
& y_{k}=h\left(x_{k}, \varphi_{k}, u_{k}\right)+v_{k}=H_{k}^{\varphi} \varphi_{k}+D_{k} i_{k}+v_{k}
\end{aligned}
$$

where $x_{k}=\left[\begin{array}{lll}S O C_{k} & V_{p, k}\end{array}\right]^{T}, \varphi_{k}=\left[\begin{array}{l}C_{c a p, k}\end{array}\right], D_{k}=$ $\left[-R_{0, k}\right], B_{k-1}=\left[-\left(\eta_{i} \Delta t / C_{c a p}\right)\left(1-e^{-1 / R_{p, k-1} C_{p, k-1}}\right) R_{p, k-1}\right]^{T}$, $F_{k-1}=\left.\left(d f\left(x_{k-1}, \widehat{\varphi}_{k \mid k-1}, u_{k-1}\right) / d x_{k-1}\right)\right|_{\widehat{x}_{k \mid k-1}}=$

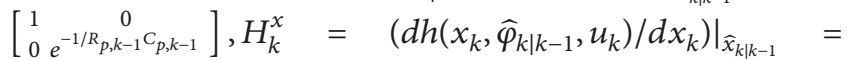
$\left[\partial V_{o c, k}\left(S C_{k}, C_{c a p, k}\right) / \partial S O C_{k}-1\right]^{T}$,

$H_{k}^{\varphi}=\left.\left(d h\left(\widehat{x}_{k \mid k-1}, \varphi_{k}, u_{k}\right) / d \varphi_{k}\right)\right|_{\hat{\varphi}_{k \mid k-1}}$, the symbols $w_{k-1}^{x}$ and $w_{k-1}^{\varphi}$ represent the state noise of the $x_{k}$ and $\varphi_{k}$, respectively, and the symbol $v_{k}$ is the measurement noise.

It is worth noting that parameter $H_{k}^{\varphi}$ of measurement equation is associated with SOC, so the $\mathrm{H}_{k}^{\varphi}$ can be expanded as follows:

$$
H_{k}^{\varphi}=\left.\frac{d h\left(\widehat{x}_{k \mid k-1}, \varphi_{k}, u_{k}\right)}{d \varphi_{k}}\right|_{\widehat{\varphi}_{k \mid k-1}}
$$

$$
\begin{aligned}
= & \left.\frac{\partial h\left(\widehat{x}_{k \mid k-1}, \varphi_{k}, u_{k}\right)}{\partial \varphi_{k}}\right|_{\widehat{\varphi}_{k \mid k-1}} \\
& +\left.\left.\frac{\partial h\left(x_{k}, \varphi_{k}, u_{k}\right)}{\partial x_{k}}\right|_{\widehat{x}_{k \mid k-1}} \frac{d x_{k}}{d \varphi_{k}}\right|_{\widehat{\varphi}_{k \mid k-1}}
\end{aligned}
$$

where $\partial h\left(x_{k}, \varphi_{k}, u_{k}\right) / \partial x_{k}=H_{k}^{x}, d x_{k} / d \varphi_{k}=\partial f\left(x_{k-1}\right.$, $\left.\varphi_{k-1}, u_{k-1}\right) / \partial \varphi_{k-1}+\left(\partial f\left(x_{k-1}, \varphi_{k-1}, u_{k-1}\right) / \partial x_{k-1}\right)\left(d x_{k-1} /\right.$ $\left.d \varphi_{k-1}\right)=\eta T i_{k-1} / C_{c a p}^{2}+F_{k-1}\left(d x_{k-1} / d \varphi_{k-1}\right)$.

Although [37] considers that capacity is not associated with measurement equation from a simplification point of view, the relationship between $\mathrm{OCV}$ and capacity cannot be ignored [47]. Inspired by [47], a new quadratic function describing capacity and coefficient of fitting function is defined as

$$
\left[\begin{array}{lllllllll}
d_{8} & d_{7} & d_{6} & d_{5} & d_{4} & d_{3} & d_{2} & d_{1} & d_{0}
\end{array}\right]^{T}=\alpha_{2}\left[\begin{array}{lllllllll}
\widehat{C}_{c a p, k}^{8} & \widehat{C}_{c a p, k}^{7} & \widehat{C}_{c a p, k}^{6} & \widehat{C}_{c a p, k}^{5} & \widehat{C}_{c a p, k}^{4} & \widehat{C}_{c a p, k}^{3} & \widehat{C}_{c a p, k}^{2} & \widehat{C}_{c a p, k}^{1} & 1
\end{array}\right]^{T}
$$

where $\alpha_{2}$ is the undetermined coefficient between capacity and OCV and is obtained via iterative method and $d_{8}-d_{0}$ are the coefficients of fitting function describing the relationship between OCV and SOC.
On the basis of the above methods,

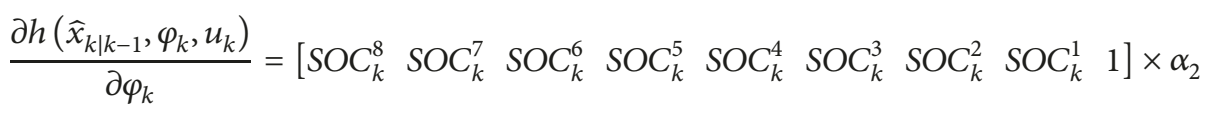

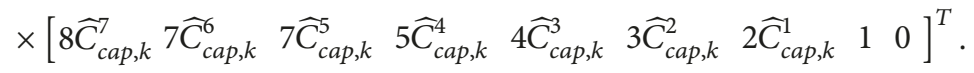

Compare to [37] and [47], in which, without consideration of the relevant degree between OCV and capacity, the $\partial h\left(\hat{x}_{k \mid k-1}, \varphi_{k}, u_{k}\right) / \partial \varphi_{k}$ can be used as fine-tuning factor for capacity error compensation (EC). With the identified ECM parameters and OCV, the battery SOC and capacity are estimated iteratively. The process of the HIF with tunable restricted factor can be seen in Table 1 , the noise covariance adaptive adjusted strategy is given in (22) and (25), the ST correction factor is listed in (30), and the general flowchart of the dual ST-AHIF based SOC and capacity joint estimation method is illustrated in Figure 3.

\section{Verification and Discussion}

The $20 \mathrm{Ah} / 24 \mathrm{~V}$ lithium-ion phosphate battery is selected as the test object based on a high precise battery test platform shown in Figure 4, which consists of a programmable temperature chamber, a connected computer, and a power battery test system (Arbin EVTS) with current (0 to 300A) and voltage (0 to $400 \mathrm{~V})$, while the voltage and current measurement error limits are both within $0.1 \%$. The computer connecting with Arbin EVTS is used to collect and store experimental data such as charge/discharge current and terminal voltage at a time interval of $1 \mathrm{~s}$. The data of two operating conditions including Dynamic Stress Test (DST) and Federal Urban Driving Schedule (FUDS) at a constant temperature $25^{\circ} \mathrm{C}$ for the battery are collected to evaluate the effectiveness of model parameters identification and state estimation method. The reference SOC should be determined accurately to evaluate the suitability of the proposed dual ST-AHIF method. The coulomb counting (CC) method with high reliability is used to calculate the reference SOC under a certain condition; that is, initial SOC is known.

In addition, the statistical indexes such as maximum absolute error (MAE) and average absolute error (AAE) are used to represent quantization performance of the identification and estimation algorithms. 


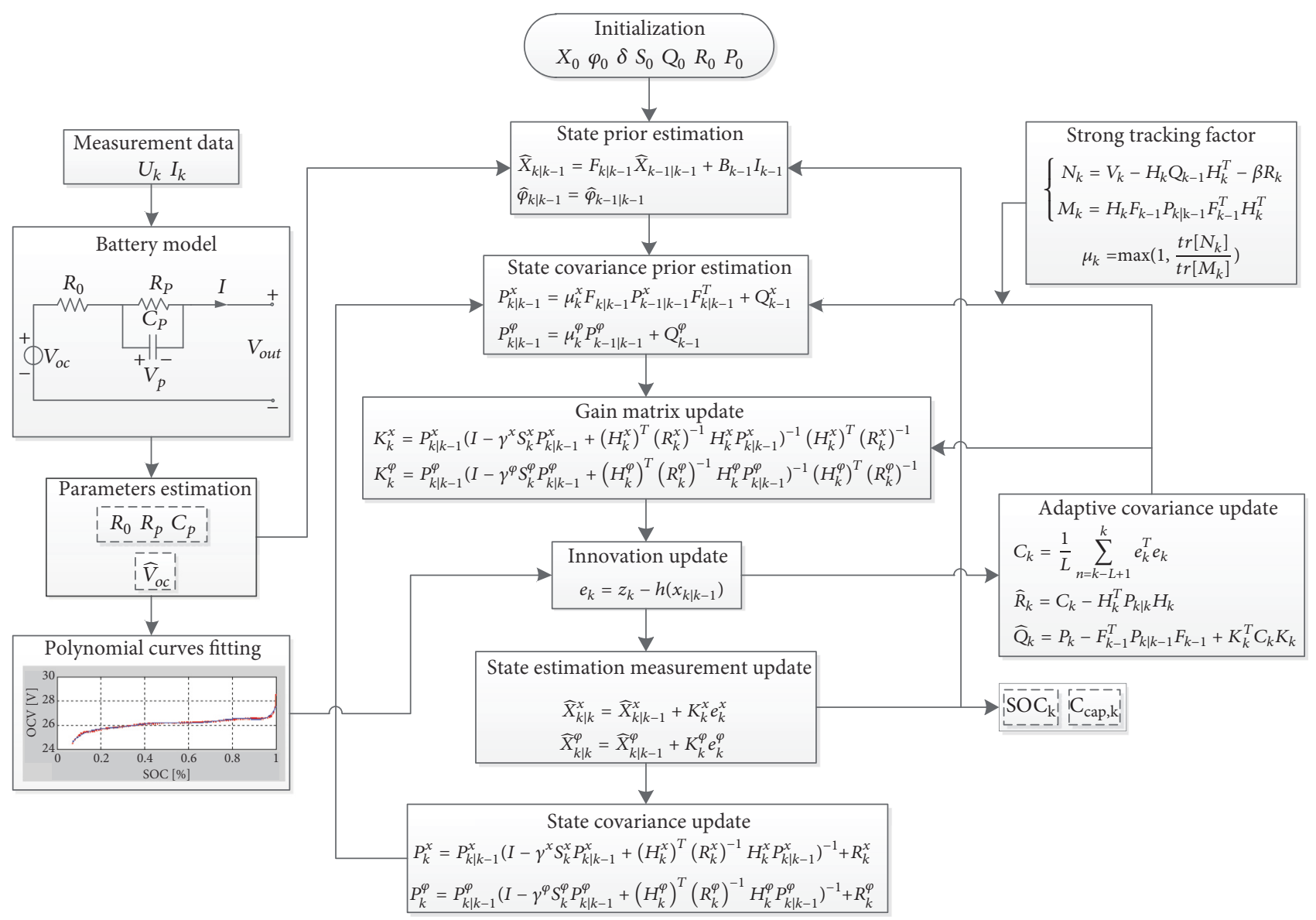

FIgURE 3: The flowchart of the dual ST-AHIF based SOC and capacity estimator.

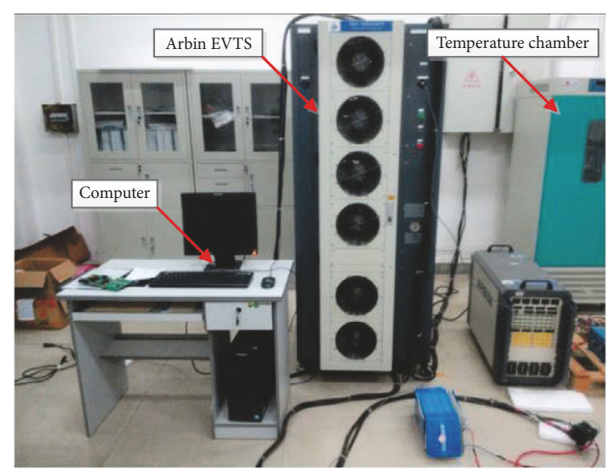

FIGURE 4: The battery test platform.

5.1. ECM Parameters Identification. The lithium-ion battery is fully charged by constant-current-constant-voltage (CCCV) method, and then it is left to rest for two hours before being discharged under two working conditions, respectively. The discharge current-time distribution of the DST and FUDS cycle, in which the duration of uninterrupted working condition is 35000 seconds, is shown in Figure 5. With the data flows of collected discharge current and terminal voltage, the VFFRLS algorithm and incremental model analysis are combined to identify the ECM parameters, OCV, and terminal voltage.

The ECM parameters are identified by using recursion procedure. To demonstrate the robustness of the VFFRLS against unreliable initial value, imprecise initial values are purposely set as follows: $R_{0}=0.05 \Omega, R_{p}=0.01 \Omega$, and $C_{p}=1000 \mathrm{~F}$. The identified ECM parameters versus time are shown in Figures 6(a)-6(b); the identification values of ECM parameters are able to converge to stable values rapidly from the unreliable initial value under the DST and FUDS cycle, respectively. Among them, the ohmic resistance $R_{0}$ and the polarization resistance $R_{P}$ exhibit similar tendencies except for a little fluctuation. Specifically, $R_{0}$ keeps a higher stability, as the ohmic resistance is equal to the ratio of terminal voltage variation to transient current when the variable current is turned off. By contrast to $R_{0}$ and $R_{P}$, the polarization capacitance $C_{P}$ varied significantly because $R_{P}$ and $C_{P}$ have direct correlation to intricate electrochemical activity. Based on the incremental analysis adaption in ECM, the VFFRLS can be robust against the varying variables such as initialization error of parameters.

With the extracted $R_{0}, R_{P}$, and $C_{P}$, the terminal voltage as ECM observation is recursively obtained in each sampling period. Figures $7(\mathrm{a})-7(\mathrm{~b})$ and $7(\mathrm{c})-7(\mathrm{~d})$ demonstrate the identified results under DST and FUDS cycle, respectively. 


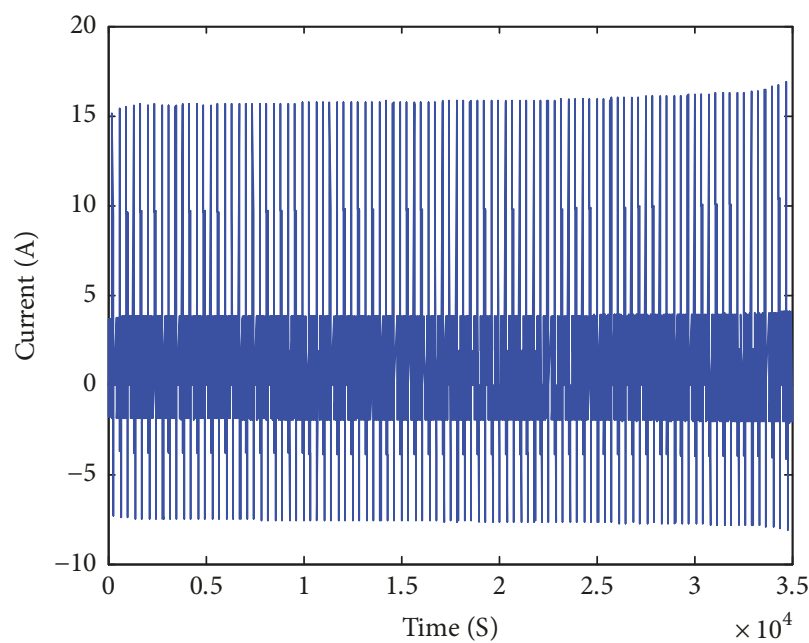

(a)

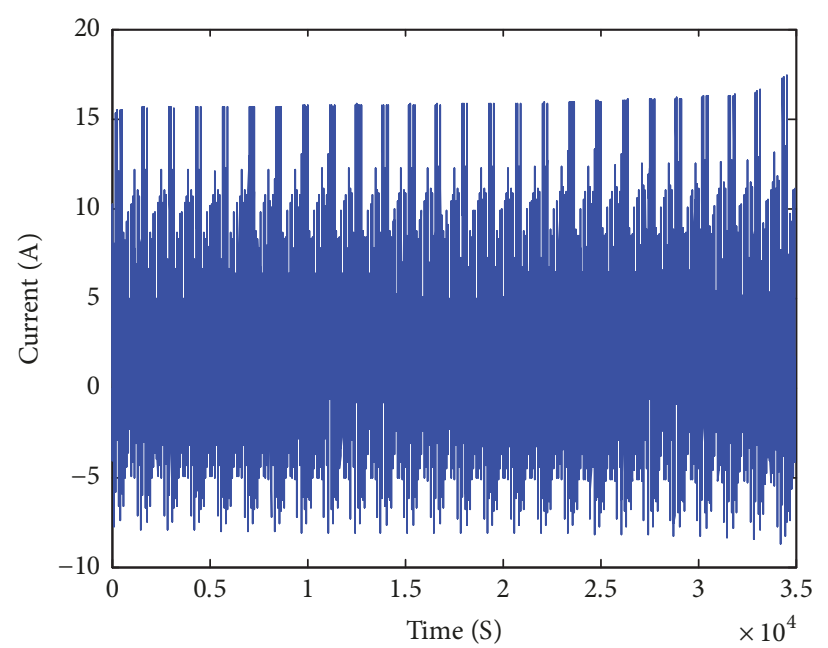

(b)

Figure 5: (a) Current under DST cycle; (b) current under FUDS cycle.
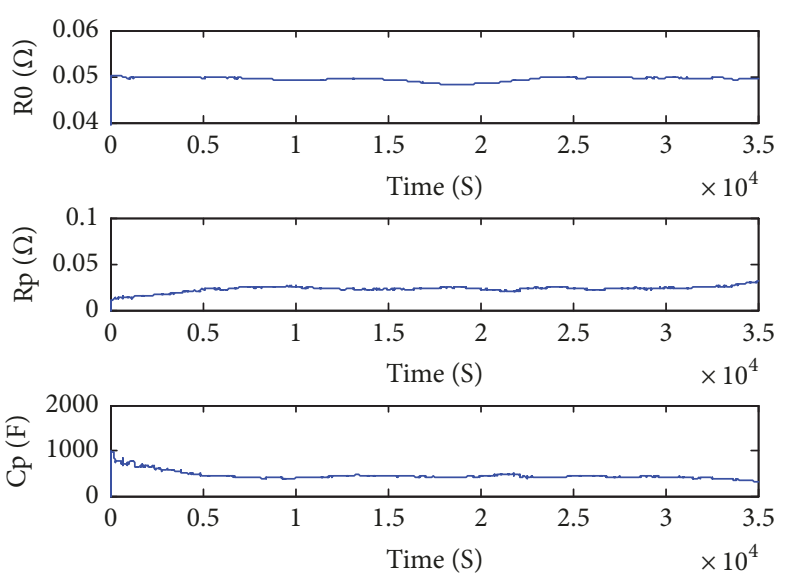

(a)
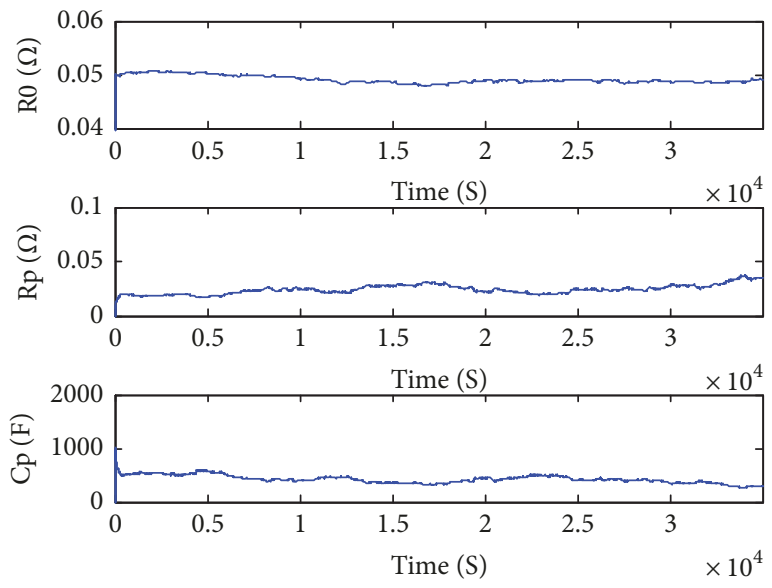

(b)

Figure 6: (a) Value of $R_{0}, R_{P}$, and $C_{P}$ under DST cycle; (b) value of $R_{0}$, $R_{p}$, and $C_{P}$ under FUDS cycle.

As can be seen, although the ECM is reduced to the oneorder Thevenin ECM, the error stabilization of the terminal voltage by single RLS and incremental VFFRLS, respectively, still has been achieved as the whole discharging process, but it has various amplitude fluctuations. Compared with original RLS, the terminal voltage estimation error of the incremental VFFRLS is obviously decreased and always keeps stability of error. As summarized in Table 2, the maximum terminal voltage error (MTVE) of the RLS is up to $0.1356 \mathrm{~V}$ and $0.1467 \mathrm{~V}$ under the DST and FUDS cycle, respectively; correspondingly, the MTVE of the incremental VFFRLS is only $0.0211 \mathrm{~V}$ and $0.0304 \mathrm{~V}$ under the DST and FUDS cycle, respectively, indicating a high identification precision. The average terminal voltage error (ATVE) of the incremental VFFRLS is only $0.0003 \mathrm{~V}$ and $0.0015 \mathrm{~V}$ under the DST and FUDS cycle, respectively, which can be neglected regarding the sensors precision. The high identification precision of terminal voltage reaffirms the validity of the ECM parameter identification method by VFFRLS and further proves the credibility of the Thevenin ECM with incremental analysis.

5.2. SOC Estimation. Based on the identified ECM parameters and terminal voltage, the SOC estimation results can be obtained with AHIF algorithm. As shown in Figure 8, the black line presents the reference SOC, while the blue line indicates the SOC estimation. Figure 8(a) shows that the estimated SOC is adaptive and capable of tracking reference SOC dynamic after a short period of time. Figure 8(b) indicates that the SOC estimation error of AHIF can not only converge to the reference SOC but also stay within $1.5 \%$ except at the beginning of discharge. It is observed that the SOC error is relatively large from $7.7 \mathrm{~h}$ to $8.6 \mathrm{~h}$ which corresponds to the SOC range between $30 \%$ and $20 \%$. Two reasons might be that (1) there exists a flat area in the OCVSOC fitting curve in that region and (2) the precision of the measurement like current and voltage directly affects the 


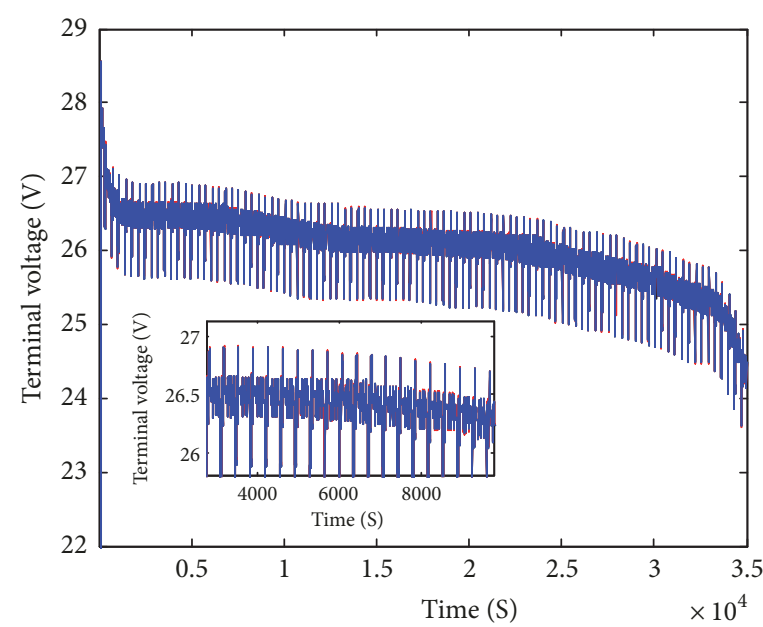

- Reference RLS

(a)

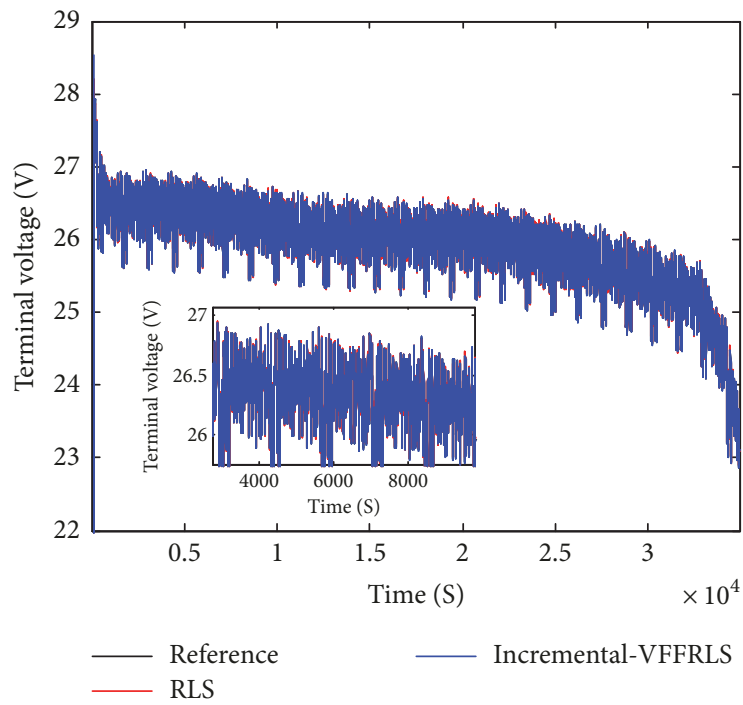

(c)

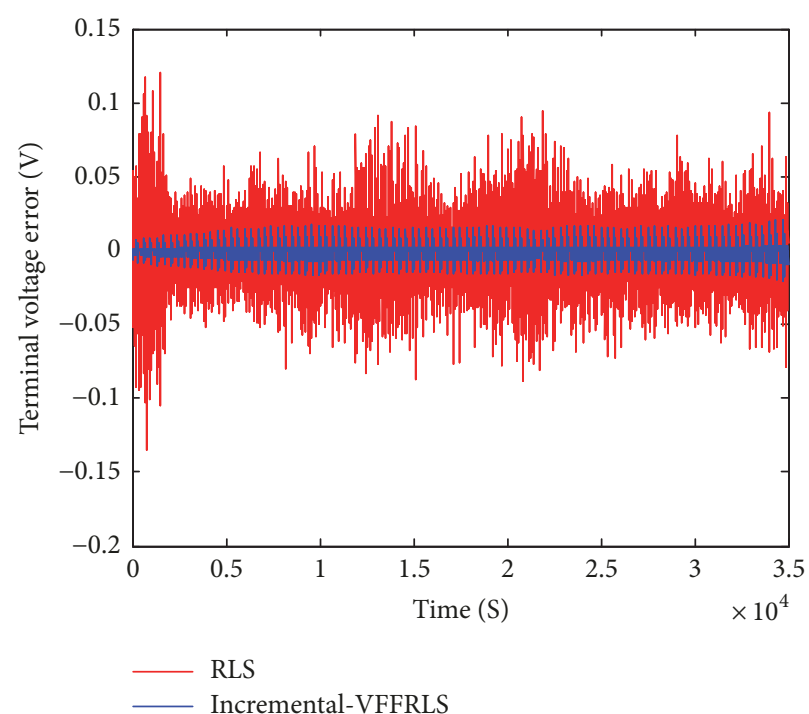

(b)

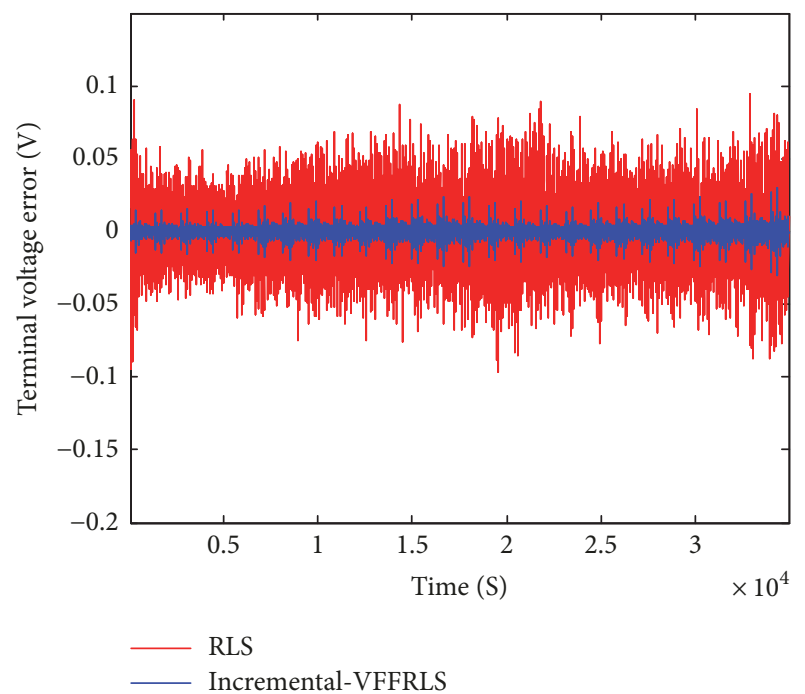

(d)

FIGURE 7: Terminal voltage comparison estimation with (a) results under DST, (b) error under DST, (c) results under FUDS, and (d) error under FUDS.

TABLE 2: Comparison for terminal voltage estimation results.

\begin{tabular}{lcccc}
\hline & RLS & RLS & $\begin{array}{c}\text { Incremental-VFFRLS } \\
\text { (DST) }\end{array}$ & $\begin{array}{c}\text { Incremental-VFFRLS } \\
\text { (FUDS) }\end{array}$ \\
\hline MTVE(V) & 0.1356 & 0.1467 & 0.0211 & 0.0304 \\
ATVE(V) & 0.0123 & 0.0141 & 0.0003 & 0.0015 \\
\hline
\end{tabular}

precision of OCV, and any small disturbances on OCV may cause larger SOC error that cannot be avoided. The AAE of AHIF is $0.4363 \%$ and the corresponding MAE is $2.1966 \%$. Although the overall results seem to be acceptable, there is scope for improvement.

To further eliminate SOC error with AHIF shown in Figure 8, the STF is incorporated into AHIF to reduce the effects of abrupt fluctuation in measurement when the estimator reaches a stable state. Figures 9(a) and 9(b) show the SOC estimation results and SOC estimation error. The black line presents the reference SOC, the blue line indicates the SOC estimation by ST-AHIF, and the red line denotes the SOC estimation by AHIF. Figure 9(a) shows that the estimated SOC by ST-AHIF can be adaptive and capable of tracking reference SOC dynamic rapidly. Figure 9(b) indicates that the SOC estimation error of ST-AHIF can not 


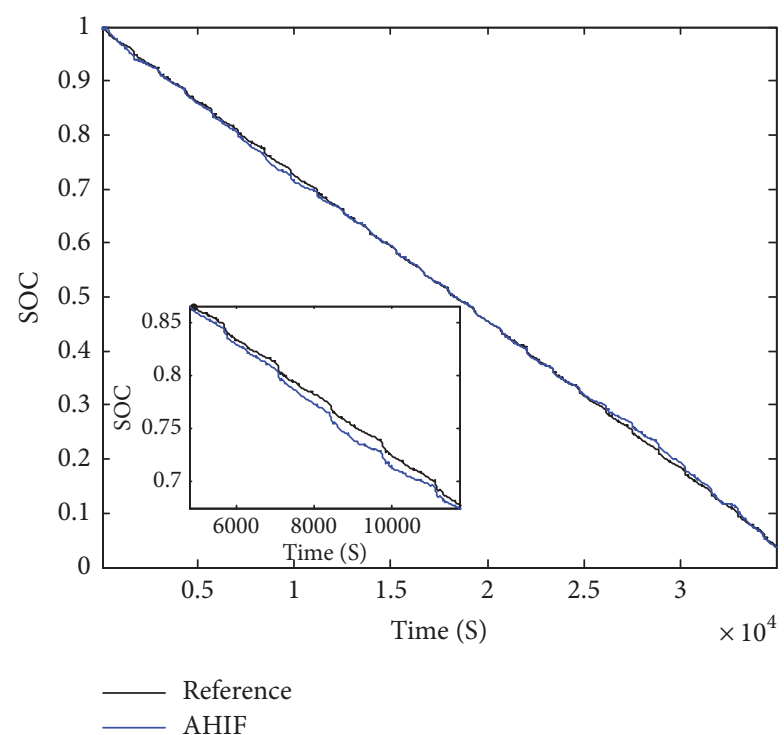

(a)

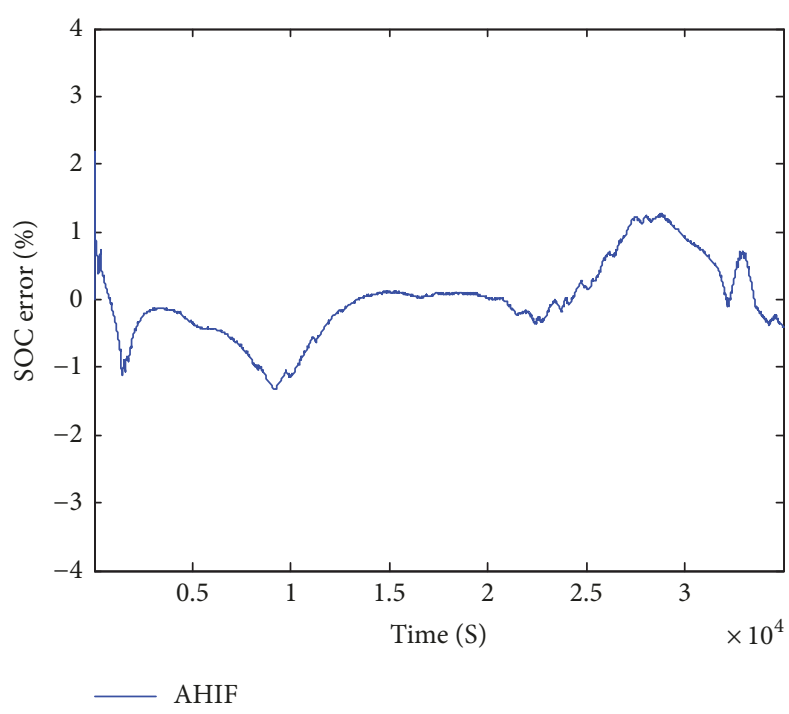

(b)

FIGURE 8: The SOC estimation results of AHIF: (a) SOC estimation; (b) SOC estimation error.

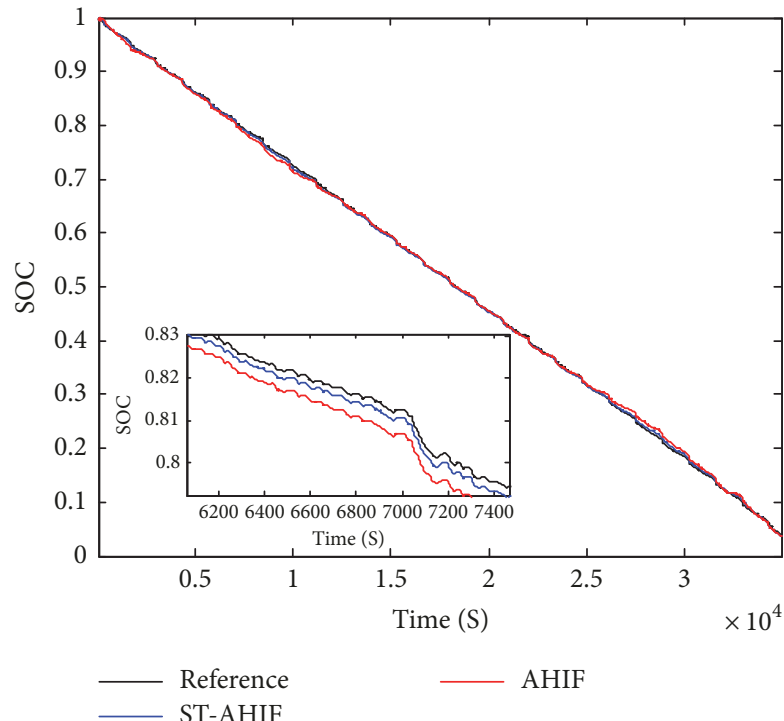

(a)

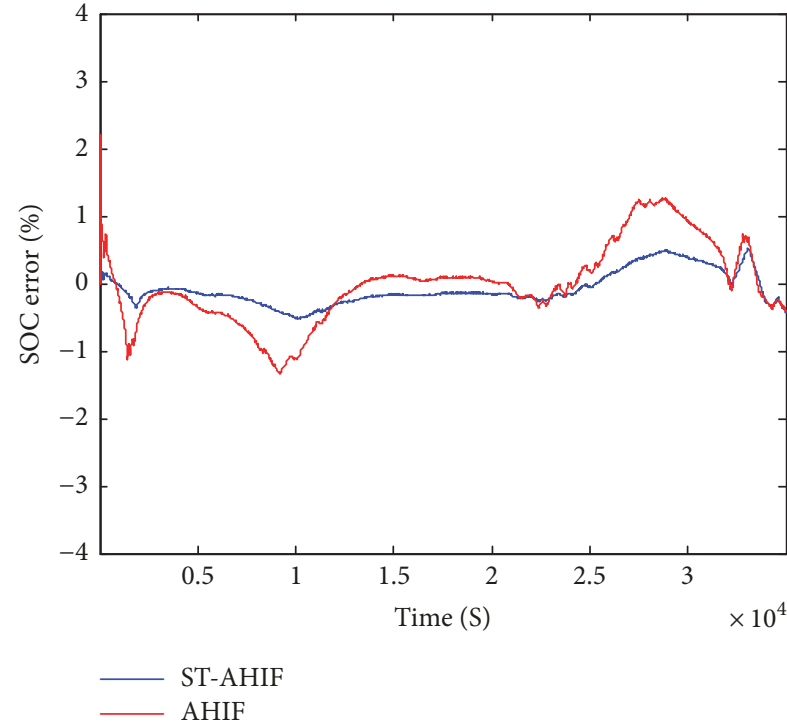

(b)

FIGURE 9: The SOC comparison results of ST-AHIF and AHIF: (a) SOC estimation; (b) SOC estimation error.

only converge to the reference SOC but also stay within $1 \%$, showing a sufficiently high accuracy. And, more remarkably, the SOC error is dramatically inhibited in those special areas that represent the relatively large SOC estimation error by the AHIF because the STF has advantageous effects on tracking sudden change and offsetting model uncertainty. The AAE of ST-AHIF is only $0.2311 \%$ and the corresponding MAE is $1.3401 \%$, which is significantly better than AHIF. Therefore, the combination of STF with AHIF has evident advantage over single AHIF in SOC estimation.

Beyond that, the commonly used AEKF is used for SOC estimation under the same condition. Figure 10 shows the SOC estimation results by comparing the ST-AHIF, AHIF, and AEKF. In Figure 10(a), the black line represents the reference SOC, the blue line indicates the SOC estimation by ST-AHIF, the red line denotes the SOC estimation by AHIF, and the green line is SOC estimation by AEKF. The SOC estimation error is plotted in Figure 10(b), where the blue line indicates the SOC estimation error by ST-AHIF, the red line denotes the SOC estimation error by AHIF, and the green line is SOC estimation error by AEKF. Due to the problem of model uncertainty and flat area in OCV-SOC fitting curve, the special area which represents the relatively large SOC estimation error still exists. In the view of this special area, AEKF and AHIF suffer the common disadvantage with similar error, both of which cannot overcome the disturbances on OCV. 
TABLE 3: Comparison for the SOC estimation results.

\begin{tabular}{lccr}
\hline Method & ST-AHIF & AHIF & AEKF \\
\hline MAE(\%) & 1.3401 & 2.1966 & 3.5675 \\
AAE(\%) & 0.2311 & 0.4363 & 0.7705 \\
\hline
\end{tabular}

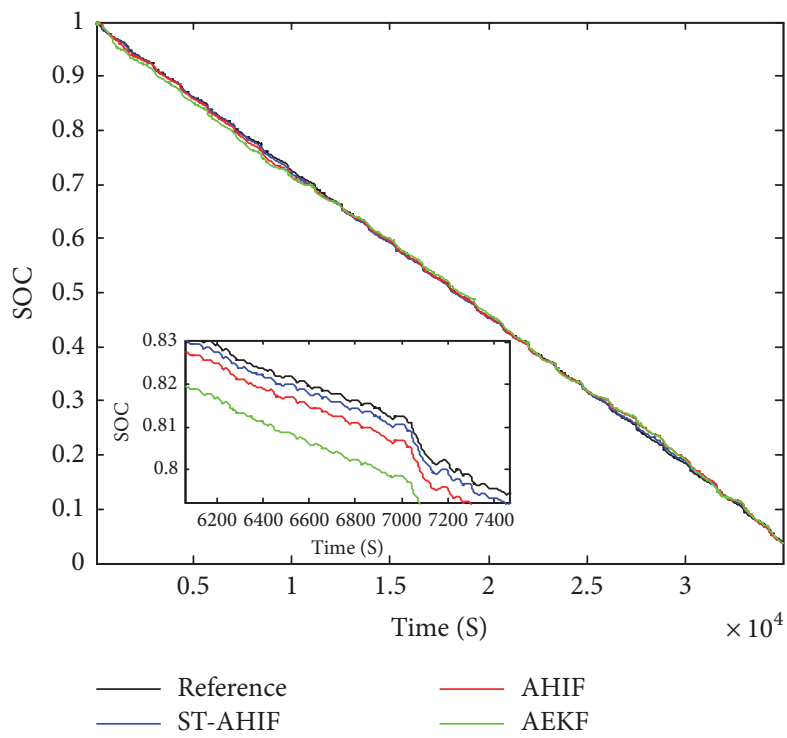

(a)

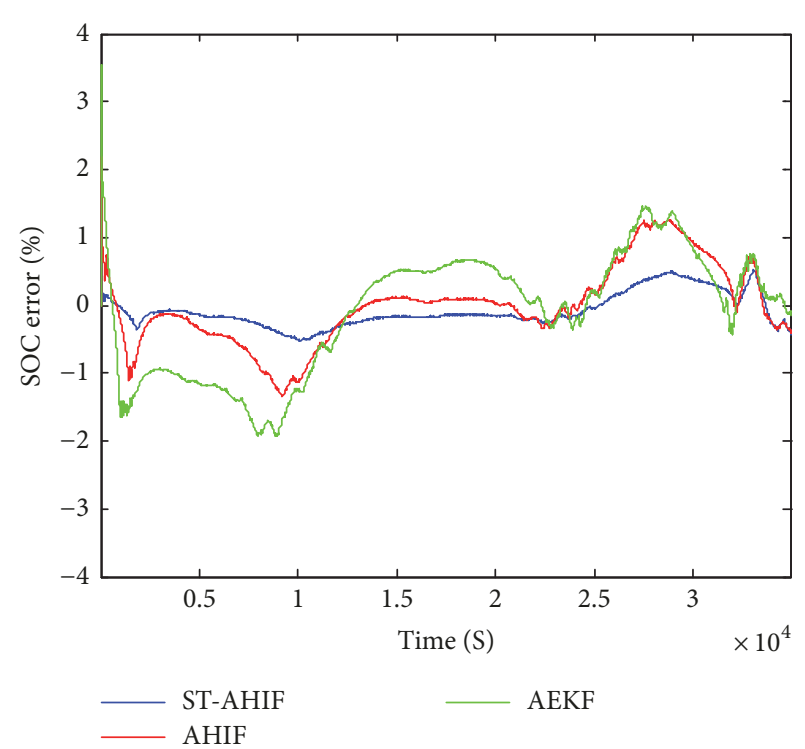

(b)

FIGURE 10: The SOC comparison results of three algorithms: (a) SOC estimation; (b) SOC estimation error.

The AAE of AEKF is $0.7705 \%$ and the corresponding MAE is $3.5675 \%$, respectively. In summary, the SOC estimation results of ST-AHIF, AHIF, and AEKF are shown as in Table 3; it is obvious that the proposed ST-AHIF is superior to AHIF and AEKF.

As the SOC estimation is reliably obtained based on known initial value, SOC initialization error may have a negative influence on the SOC estimation. To evaluate the possible negative effect, three levels of initialization error on SOC from $10 \%, 20 \%$, and $30 \%$ are used for the proposed ST-AHIF, respectively. The estimation results and estimation error of SOC are shown in Figure 11. The black line represents the reference SOC, the blue line indicates the SOC estimation with $10 \%$ initialization error, the red line denotes the SOC estimation with $20 \%$ initialization error, and the green line is SOC estimation with $30 \%$ initialization error. It is clear that the global SOC estimation error with three levels of initialization error is increased throughout the whole discharge process. It is obvious that the special area which represents the relatively large SOC estimation is observed. However, the estimation error is still within reasonable range; the convergence and accuracy have not been markedly weakened. Although the SOC estimation error is relatively large at the beginning of discharging process when SOC is initialized with error of $10 \%, 20 \%$, and $30 \%$, respectively, the estimated SOC can track reference SOC rapidly and closely until the end of discharging. In summary, the SOC estimation results with influence of three SOC initialization errors are shown as in Table 4.
TABLE 4: Comparison for the SOC estimation results with initialization error

\begin{tabular}{lccc}
\hline ST-AHIF & $90 \%$ & $80 \%$ & $70 \%$ \\
\hline AAE(\%) & 0.5441 & 0.6684 & 0.7336 \\
\hline
\end{tabular}

\subsection{Capacity Estimation}

5.3.1. Evaluation of ST-AHIF and AHIF. With the FUDS data at constant temperature $25^{\circ} \mathrm{C}$, the capacity estimation results by ST-AHIF and AHIF, respectively, in which the relationship between OCV and capacity has not been taken into consideration, are plotted in Figure 12. The black line represents the capacity reference value, the red line is the capacity estimation results by AHIF, and the blue line is the capacity estimation results by ST-AHIF. Figures 12 (b) and 12(d) show that no matter the capacity estimated by AHIF or ST-AHIF, it has similar estimation trend. However, the STAHIF has higher estimation accuracy than the AHIF. Due to the lacking of EC, both of the two capacity estimations have relatively large error. Although the ST-AHIF has advantages on the issue of restraining model uncertainty and abrupt fluctuation compared with AHIF, the error tends to increase, particularly the error of capacity estimation close to $4 \%$ in the special areas corresponding to larger SOC estimation error, which is beyond the acceptable range. Therefore, it is necessary to improve capacity estimation precision through adding appropriate EC into ST-AHIF. 


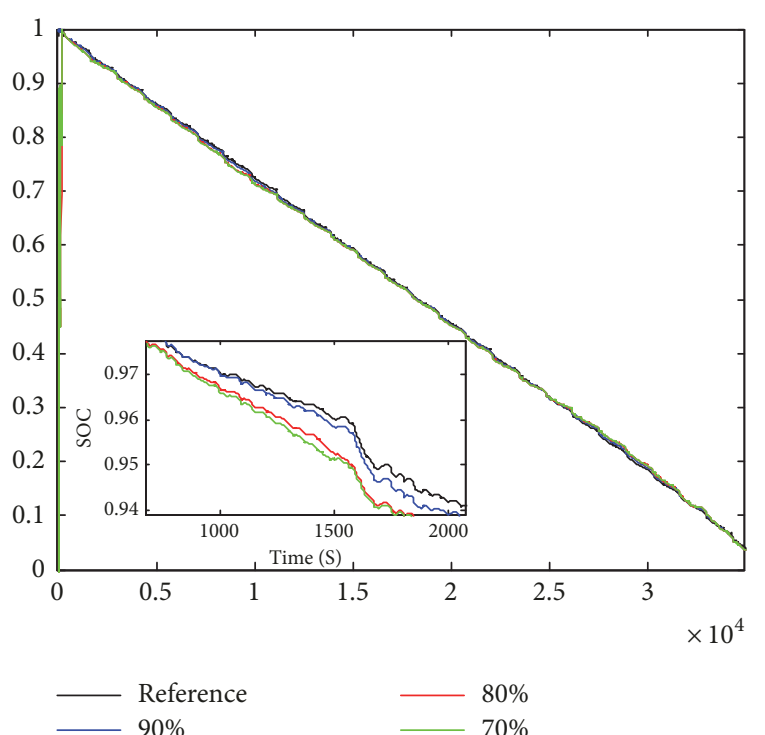

(a)

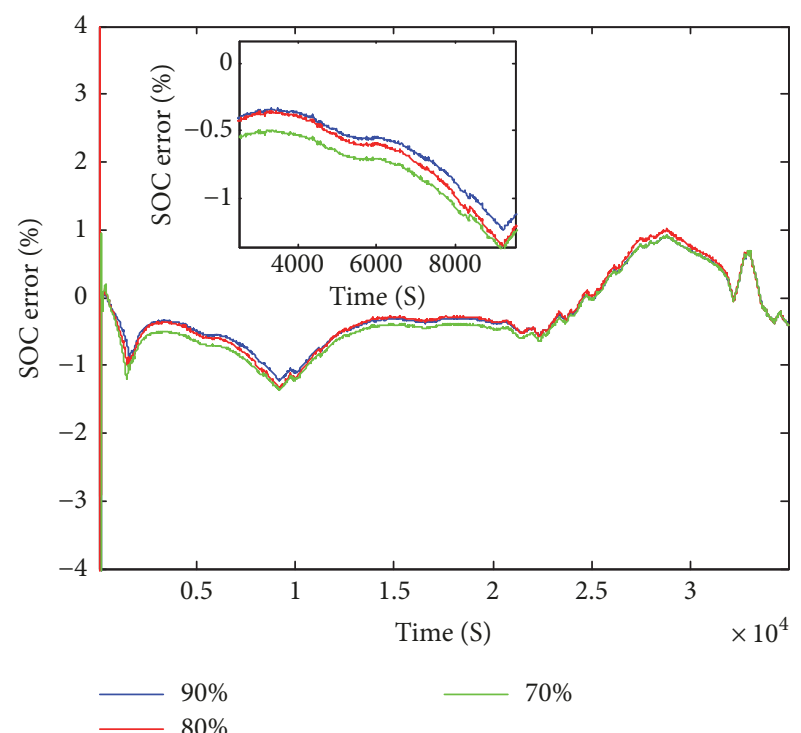

(b)

FIGURE 11: The SOC comparison results with different SOC initialization error: (a) SOC estimation; (b) SOC estimation error.

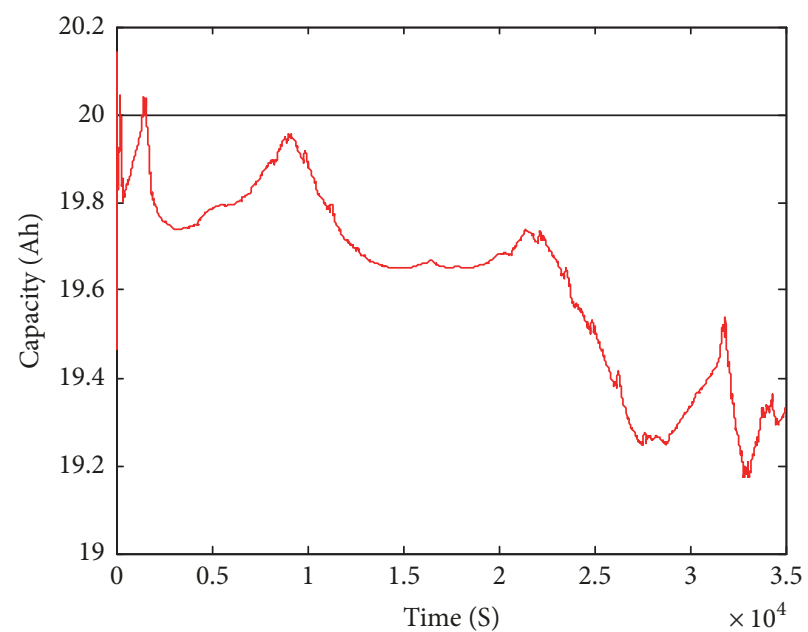

Reference

(a)

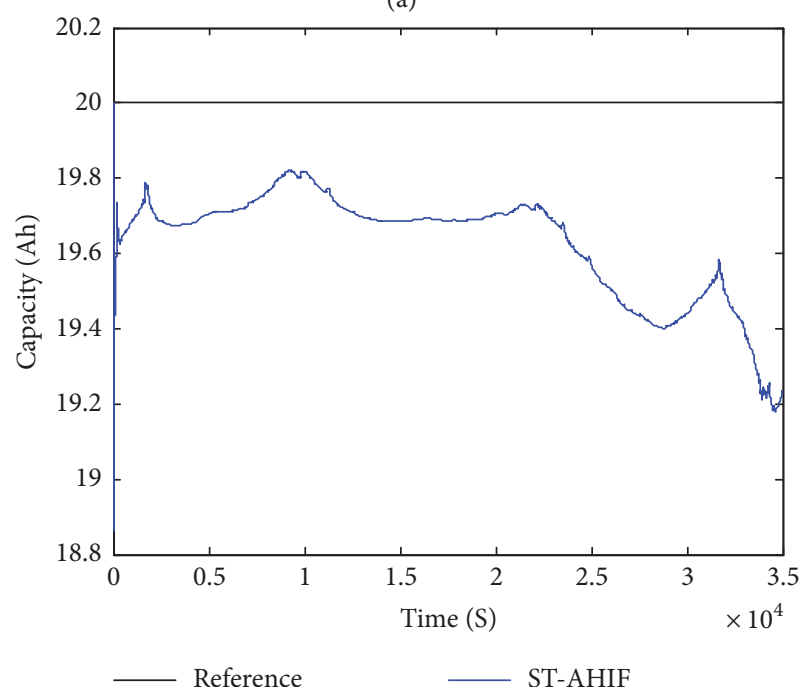

(c)

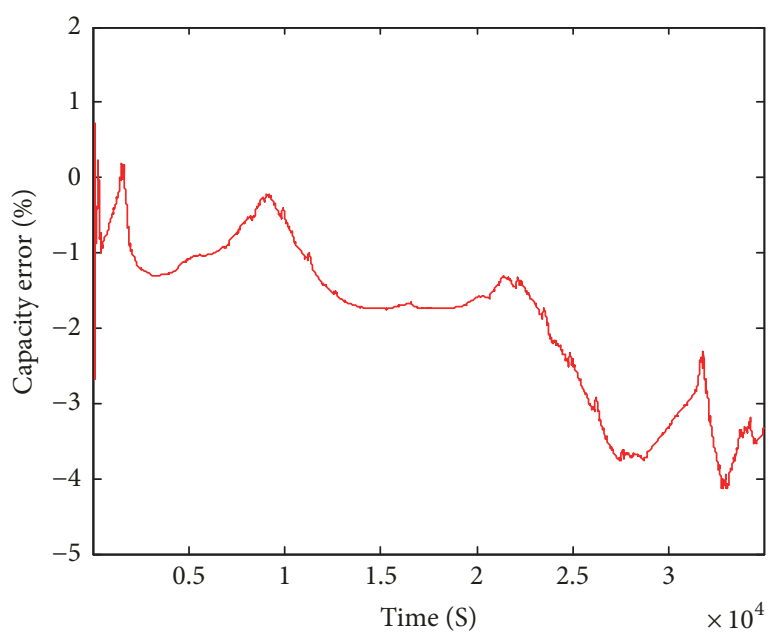

AHIF

(b)

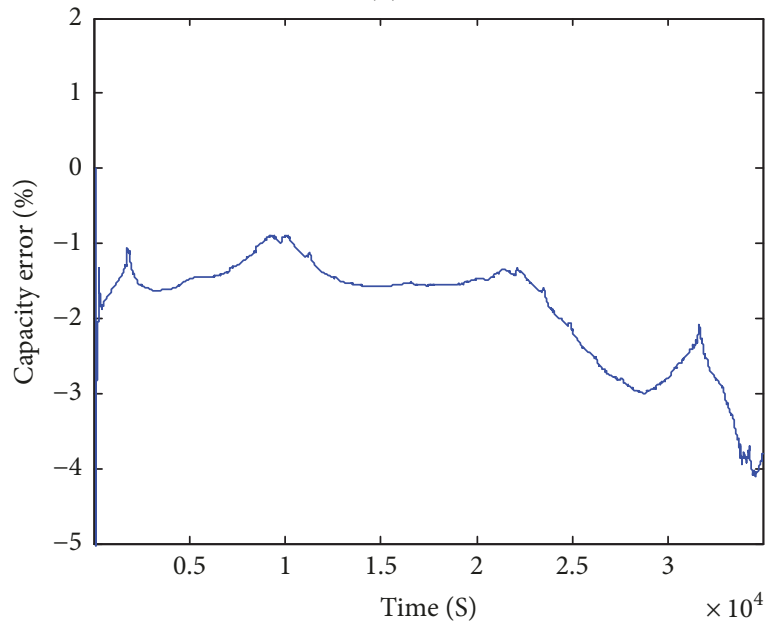

ST-AHIF

(d)

FIGURE 12: The comparison results without EC: (a) capacity estimation by AHIF; (b) corresponding estimation error by AHIF; (c) capacity estimation by ST-AHIF; (d) corresponding estimation error by ST-AHIF. 


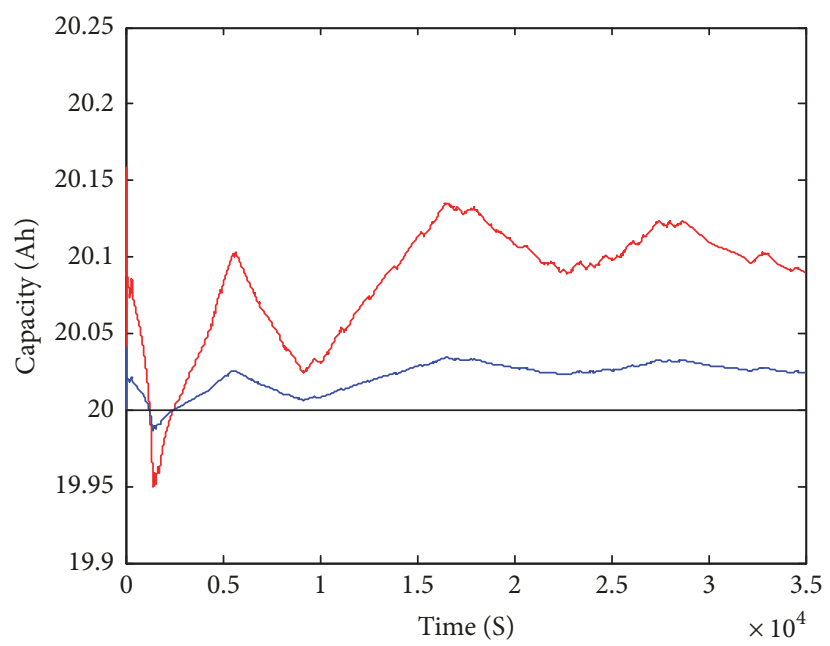

AHIF-Ref.[47] EC
AHIF-Improved EC

(a)

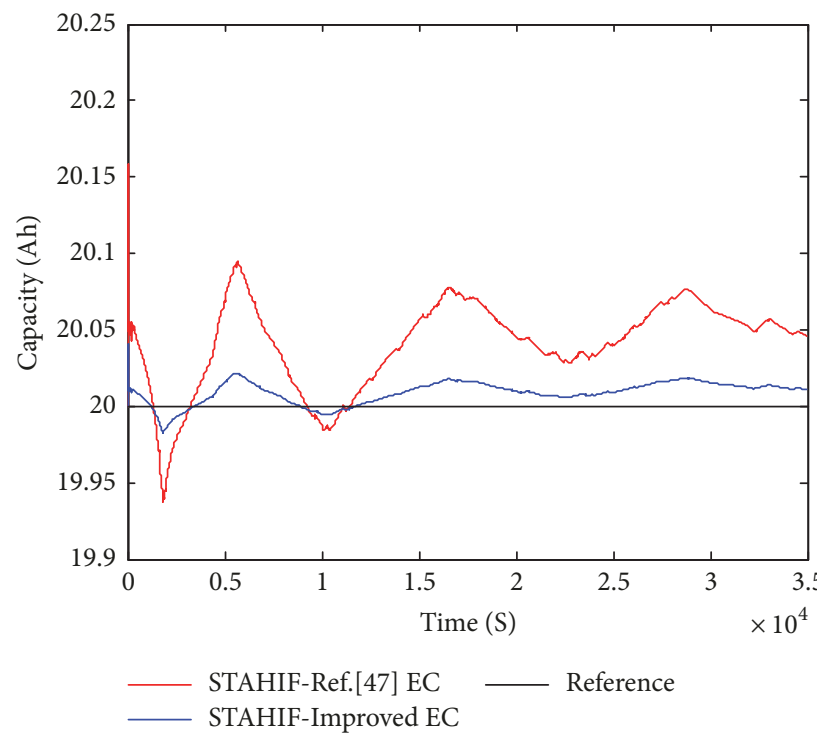

(c)

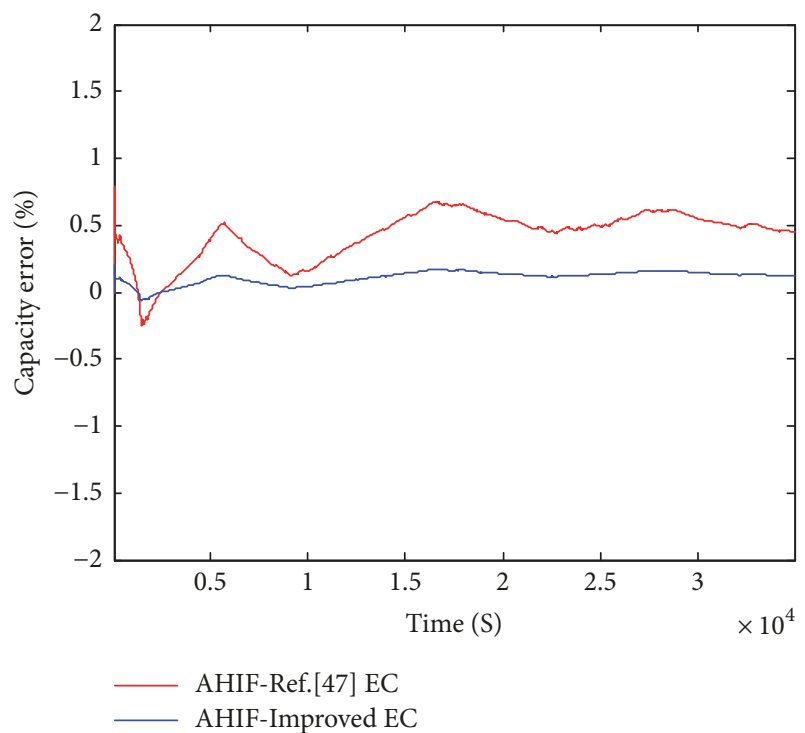

(b)

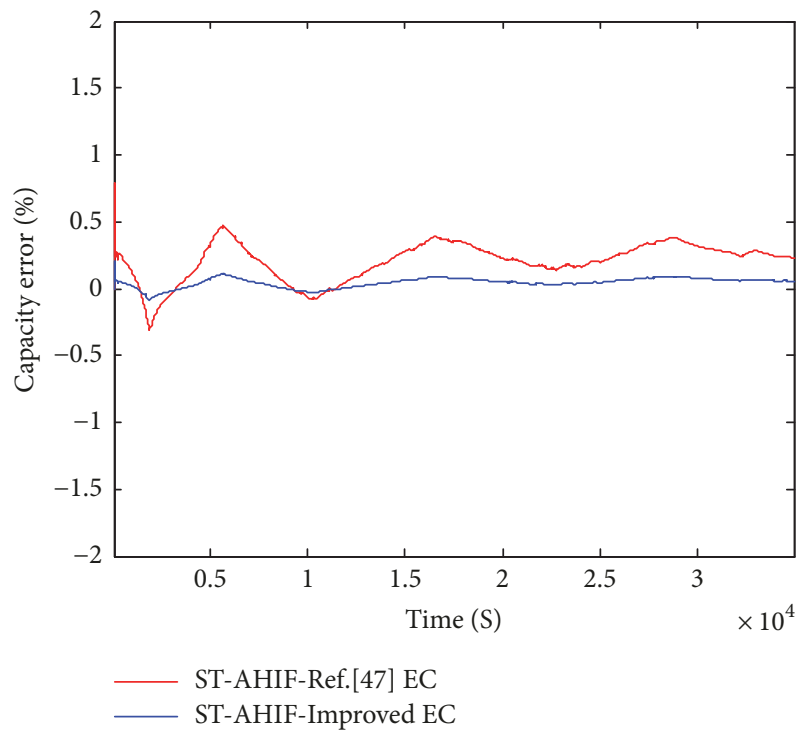

(d)

FIGURE 13: The comparison results with two EC: (a) capacity estimation by AHIF; (b) corresponding estimation error by AHIF; (c) capacity estimation by ST-AHIF; (d) corresponding estimation error by ST-AHIF.

5.3.2. Evaluation of Improved EC. In order to verify the effect of improved EC, the capacity estimation results of the STAHIF and the AHIF are plotted in Figure 13, where the two different EC are introduced to compare the estimation performance. Figures 13(a)-13(b) indicate the capacity estimation results through AHIF with EC in [47] and improved EC, respectively. Figures 13(c)-13(d) show the capacity estimation results by ST-AHIF with two EC, respectively. The black line represents the capacity reference value, the red line is the capacity estimation results with EC in [47], and the blue line is the capacity estimation results with improved EC. Figures 13(a) and 13(c) show that the capacity estimations with EC can both fast converge to the reference capacity no matter the EC in [47] or improved EC. Figures 13(b) and 13(d) show that estimation error is significantly lower than that in Figures 12(b) and 12(d). Among them, the capacity estimation error obtained by EC in [47] can remain within 1\% while the capacity estimation error got by improved EC is kept in $0.5 \%$ no matter whether in AHIF or ST-AHIF. Thus it can be seen that introduction of EC is effective for improving dynamic capacity estimation approach, and especially the relatively big capacity estimation error is significantly reduced in special areas corresponding to relatively big SOC estimation error. By comparing these two EC in Figures 13(b) and 13(d), the capacity estimation error calculated with improved EC has smaller fluctuation and higher precision than estimation error with EC in [47]. The above results analyses indicate that, under the same EC condition, the capacity 
TABLE 5: Comparison for the capacity results.

\begin{tabular}{lccc}
\hline Method & ST-AHIF & AHIF & AEKF \\
\hline MAE ([47]) & $0.7915 \%$ & $0.9050 \%$ & $1.6812 \%$ \\
MAE (Improved) & $0.2057 \%$ & $0.2511 \%$ & $0.4228 \%$ \\
AAE ([47]) & $0.1697 \%$ & $0.4326 \%$ & $1.0933 \%$ \\
AAE (Improved) & $0.0514 \%$ & $0.1113 \%$ & $0.2778 \%$ \\
\hline
\end{tabular}

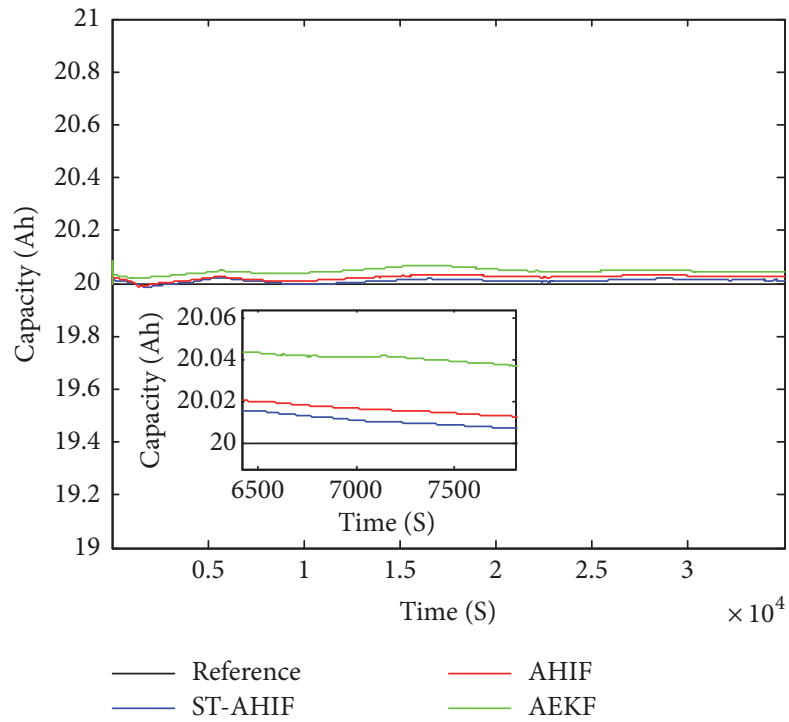

(a)

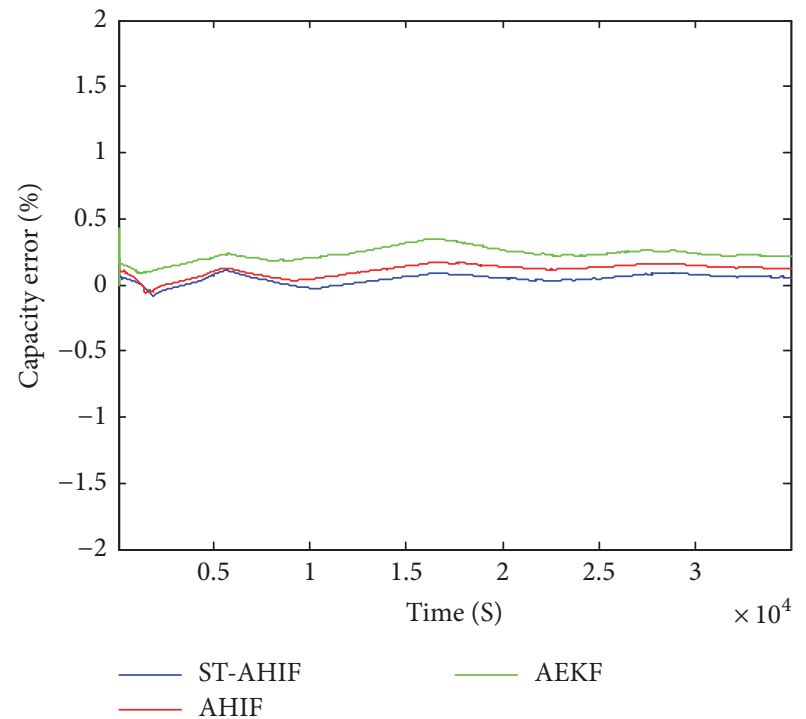

(b)

FIGURE 14: The comparison results with improved EC by three algorithms: (a) capacity estimation; (b) capacity estimation error.

estimation precision by ST-AHIF is higher than that by AHIF.

Similarly, Figure 14(a) shows the reference and estimated capacity results by AEKF, AHIF, and ST-AHIF with improved EC, and Figure 14(b) shows their capacity estimation error. The black line represents the capacity reference value, the green line is the capacity estimation value by AEKF, the red line denotes the capacity estimation value by AHIF, and the blue line is the capacity estimation value by ST-AHIF. Figure 14(a) shows that the three algorithms can quickly converge to the reference capacity. Figure 14(b) indicates that the capacity estimation error obtained from the AEKF goes beyond $0.4 \%$, and the capacity estimation error obtained from the AHIF can keep in $0.26 \%$ while the capacity estimation error obtained from the ST-AHIF can keep in $0.21 \%$. It can be concluded that the ST-AHIF has smaller estimation error with improved EC than the AHIF and AEKF. Compared with Figures 13 and 14, the improved EC is suitable for the three algorithms and is superior to [47]. The MAE and AAE based on two EC are summarized in Table 5 .

\section{Conclusion}

The battery model parameters identification and states estimation suffer from model uncertainties and abrupt state instability, which results in poor convergence and low precision. To solve this problem, this paper proposes a dual ST-AHIF algorithm for SOC and capacity estimation based on real-time identified model parameters. The incrementalanalysis-based one-order Thevenin ECM combined with VFFRLS algorithm is utilized to identify model parameters; meanwhile the AHIF including STF is used to estimate SOC and capacity with consideration of relevant degree between OCV and capacity. Therefore, the proposed method for SOC and capacity estimation with fast convergence and high precision can effectively reduce the impact of model uncertainties and abrupt state instability. A high precision experimental platform has been established to gather reliable data of charge/discharge current and terminal voltage. Two typical operation conditions (DST and FUDS) are adopted to evaluate the performance of parameters identification and state estimation. The simulation results indicate the favorable estimation performances whose MAE of SOC is $1.3401 \%$ and AAE of SOC is $0.2311 \%$, while MAE of capacity is $0.2057 \%$ and AAE of capacity is $0.0514 \%$. The comparison with AHIF and AEKF further validates the superiority of the proposed integrated method in terms of precision and robustness.

\section{Nomenclature}

\section{Abbreviations and Notations}

SOC: State of charge

BMS: Battery management system

EVs: Electric vehicles

OCV: Open-circuit voltage 
KF: Kalman filter

EKF: Extended Kalman filter

UKF: Unscented Kalman filter

HIF: $\mathrm{H}$ infinity filter

ECM: Equivalent circuit model

$\mathrm{R}_{0}$ : Ohmic resistance

Rp: Polarization resistance

Cp: Polarization capacitance

Voc: Open-circuit voltage

Vp: Polarization voltage across $\mathrm{Cp}$

Vout: Terminal voltage

Voc: Open-circuit voltage

$\mathrm{I}_{\mathrm{k}}$ : Current at time $\mathrm{k}$

$\mathrm{e}_{\mathrm{k}}$ : Innovation at time $\mathrm{k}$

$\mathrm{a}_{\mathrm{i}} / \mathrm{b}_{\mathrm{i}}$ : Coefficient of parameters

$\Delta V_{\text {out }}$ : Change of terminal voltage

$\theta: \quad$ Identified parameters

$\phi$ : Measurements

$\mathrm{d}_{\mathrm{i}}: \quad$ Polynomial coefficients

$\gamma$ : $\quad$ Restricted factor

$\alpha_{1}$ : $\quad$ Corrected factor

$\beta_{1}$ : Coefficient associated with experiments

$V_{k}$ : Residual covariance matrix

$\varepsilon_{k}: \quad$ Output residuals

$\rho$ : Forgetting factor

$\mu_{k}:$ Fading factor

$\mathrm{C}_{\text {cap }}$ : Capacity

$\mathrm{R}_{\mathrm{k}}$ : Observation noise covariance

$\mathrm{Q}_{\mathrm{k}}$ : State noise covariance

$\mathrm{x}_{\mathrm{k}}$ : Estimated state including SOC

$\varphi_{k}$ : Estimated state including capacity

$\alpha_{2}$ : Correlation coefficient.

\section{Data Availability}

The data used to support the findings of this study are included within the article and are available from the corresponding author on reasonable request.

\section{Conflicts of Interest}

The authors declare no conflicts of interest.

\section{Authors' Contributions}

Zheng Liu and Xuanju Dang proposed the original idea. Zheng Liu designed a novel algorithm. Xuanju Dang and Zheng Liu performed and analyzed the experiments together. Zheng Liu wrote the original manuscript. Xuanju Dang revised the final manuscript.

\section{Acknowledgments}

This work was financially supported by the National Natural Science Foundation Project of China (61863007, 61863008), Information Science Project of Guangxi Experiment Center (20130110), Guangxi Natural Science Foundation (2015GXNSFAA139297, 2016GXNSFDA380001), and
Fundamental Ability Enhancement Project for Young and Middle-Aged University Teachers in Guangxi Province (2017KY0865).

\section{References}

[1] G. Ren, G. Ma, and N. Cong, "Review of electrical energy storage system for vehicular applications," Renewable \& Sustainable Energy Reviews, vol. 41, pp. 225-236, 2015.

[2] F. Sun, R. Xiong, and H. He, "A systematic state-of-charge estimation framework for multi-cell battery pack in electric vehicles using bias correction technique," Applied Energy, pp. 1399-1409, 2014.

[3] Y. Wang, Z. Chen, and C. Zhang, "On-line remaining energy prediction: A case study in embedded battery management system," Applied Energy, vol. 194, pp. 688-695, 2017.

[4] Z. Xie, S. Ellis, W. Xu, D. Dye, J. Zhao, and Y. Wang, "A novel preparation of core-shell electrode materials via evaporationinduced self-assembly of nanoparticles for advanced Li-ion batteries," Chemical Communications, vol. 51, no. 81, pp. 15000 15003, 2015.

[5] X. Lai, Y. Zheng, and T. Sun, "A comparative study of different equivalent circuit models for estimating state-of-charge of lithium-ion batteries," Electrochimica Acta, vol. 259, pp. 566$577,2018$.

[6] H. Wang, M. M. Ur Rehman, M. Evzelman, and R. Zane, "SIMULINK based hardware-in-the-loop rapid prototyping of an electric vehicle battery balancing controller," Control and Modeling for Power Electronics, pp. 1-16, 2015.

[7] C. Weng, J. Sun, and H. Peng, "A unified open-circuit-voltage model of lithium-ion batteries for state-of-charge estimation and state-of-health monitoring," Journal of Power Sources, vol. 258, pp. 228-237, 2014.

[8] F. Zheng, Y. Xing, J. Jiang, B. Sun, J. Kim, and M. Pecht, "Influence of different open circuit voltage tests on state of charge online estimation for lithium-ion batteries," Applied Energy, vol. 183, pp. 513-525, 2016.

[9] M. Zi-Lin, M. Xao-Jian, W. Jun-Xi, Q. Jia-Xi, and Z. Bin, "Research on SOC estimated strategy of $\mathrm{Ni} / \mathrm{MH}$ battery used for hybrid electric vehicle," in Proceedings of the The 2008 IEEE Vehicle Power and Propulsion Conference (VPPC), pp. 1-4, 2008.

[10] S. N. Kong, C. S. Moo, Y. P. Chen, and Y. C. Hsieh, "Enhanced coulomb counting method for estimating state-of-charge and state-of-health of lithium-ion batteries," Applied Energy, vol. 86, no. 9, pp. 1506-1511, 2009.

[11] L. Kang, X. Zhao, and J. Ma, "A new neural network model for the state-of-charge estimation in the battery degradation process," Applied Energy, vol. 121, pp. 20-27, 2014.

[12] X. Dang, L. Yan, H. Jiang, X. Wu, and H. Sun, "Open-circuit voltage-based state of charge estimation of lithium-ion power battery by combining controlled auto-regressive and moving average modeling with feedforward-feedback compensation method," International Journal of Electrical Power \& Energy Systems, vol. 90, pp. 27-36, 2017.

[13] C. Burgos, D. Sáez, M. E. Orchard, and R. Cárdenas, "Fuzzy modeling for the state-of-charge estimation of lead-acid batteries," Journal of Power Sources, vol. 274, pp. 355-366, 2015.

[14] H. Sheng and J. Xiao, "Electric vehicle state of charge estimation: Nonlinear correlation and fuzzy support vector machine," Journal of Power Sources, vol. 281, pp. 131-137, 2015. 
[15] V. Klass, M. Behm, and G. Lindbergh, "Capturing lithium-ion battery dynamics with support vector machine-based battery model," Journal of Power Sources, vol. 298, pp. 92-101, 2015.

[16] T. Feng, L. Yang, X. Zhao, H. Zhang, and J. Qiang, "Online identification of lithium-ion battery parameters based on an improved equivalent-circuit model and its implementation on battery state-of-power prediction," Journal of Power Sources, vol. 281, pp. 192-203, 2015.

[17] Y. Li, L. Wang, C. Liao, L. Wang, and D. Xu, "Recursive modeling and online identification of lithium-ion batteries for electric vehicle applications," Science China Technological Sciences, vol. 57, no. 2, pp. 403-413, 2014.

[18] F. Yang, Y. Xing, D. Wang, and K.-L. Tsui, "A comparative study of three model-based algorithms for estimating state-of-charge of lithium-ion batteries under a new combined dynamic loading profile," Applied Energy, vol. 164, pp. 387-399, 2016.

[19] P. Tagade, K. S. Hariharan, P. Gambhire et al., "Recursive Bayesian filtering framework for lithium-ion cell state estimation," Journal of Power Sources, vol. 306, pp. 274-288, 2016.

[20] Y. Li, C. Wang, and J. Gong, "A combination Kalman filter approach for State of Charge estimation of lithium-ion battery considering model uncertainty," Energy, vol. 109, pp. 933-946, 2016.

[21] Y. Li, C. Wang, and J. Gong, "A multi-model probability SOC fusion estimation approach using an improved adaptive unscented Kalman filter technique," Energy, vol. 141, pp. 14021415, 2017.

[22] H. K. Sahoo and P. K. Dash, "Robust estimation of power quality disturbances using unscented H1 filter," Electrical Power and Energy Systems, vol. 73, pp. 438-447, 2015.

[23] Y. Zhang, R. Xiong, H. He, and W. Shen, "Lithium-Ion Battery Pack State of Charge and State of Energy Estimation Algorithms Using a Hardware-in-The-Loop Validation," IEEE Transactions on Power Electronics, vol. 32, no. 6, pp. 4421-4431, 2017.

[24] C. Chen, R. Xiong, and W. Shen, "A lithium-ion battery-inthe-loop approach to test and validate multi-scale dual $\mathrm{H}$ infinity filters for state of charge and capacity estimation," IEEE Transactions on Power Electronics, vol. 33, no. 1, pp. 332-342, 2018.

[25] M. Charkhgard and M. H. Zarif, "Design of adaptive $H_{\infty}$ filter for implementing on state-of-charge estimation based on battery state-of-charge-varying modeling," IET Power Electronics, vol. 8, no. 10, pp. 1825-1833, 2015.

[26] C. Lin, H. Mu, R. Xiong, and W. Shen, "A novel multi-model probability battery state of charge estimation approach for electric vehicles using $\mathrm{H}$-infinity algorithm," Applied Energy, vol. 166, pp. 76-83, 2016.

[27] C. Lin, H. Mu, R. Xiong, and J. Cao, "Multi-model probabilities based state fusion estimation method of lithium-ion battery for electric vehicles: State-of-energy," Applied Energy, vol. 194, pp. 560-568, 2017.

[28] C. Zhang, W. Allafi, Q. Dinh, P. Ascencio, and J. Marco, “Online estimation of battery equivalent circuit model parameters and state of charge using decoupled least squares technique," Energy, vol. 142, pp. 678-688, 2018.

[29] H. Dai, T. Xu, L. Zhu, X. Wei, and Z. Sun, "Adaptive model parameter identification for large capacity Li-ion batteries on separated time scales," Applied Energy, vol. 184, pp. 119-131, 2016.
[30] Y. Wang, C. Zhang, and Z. Chen, "On-line battery state-ofcharge estimation based on an integrated estimator," Applied Energy, vol. 185, pp. 2026-2032, 2017.

[31] B. Xia, H. Wang, M. Wang, W. Sun, Z. Xu, and Y. Lai, "A new method for state of charge estimation of lithium-ion battery based on strong tracking cubature kalman filter," Energies, vol. 8, no. 12, pp. 13458-13472, 2015.

[32] D. Li, J. Ouyang, H. Li, and J. Wan, "State of charge estimation for LiMn2O4 power battery based on strong tracking sigma point Kalman filter," Journal of Power Sources, vol. 279, pp. 439449, 2015.

[33] Z. Yu, Y. Guo, and H. Wang, "Research on state of charge estimation of Li-ion battery based on SCKF-STF," Electric Machines and Control, vol. 17, pp. 70-76, 2013.

[34] W. Waag, C. Fleischer, and D. U. Sauer, "Critical review of the methods for monitoring of lithium-ion batteries in electric and hybrid vehicles," Journal of Power Sources, vol. 258, pp. 321-339, 2014.

[35] S. Grolleau, A. Delaille, H. Gualous et al., "Calendar aging of commercial graphite/LiFePO4 cell-Predicting capacity fade under time dependent storage conditions," Journal of Power Sources, vol. 255, pp. 450-458, 2014.

[36] X. Tang, X. Mao, J. Lin, and B. Koch, "Capacity estimation for liion batteries," in Proceedings of the American Control Conference (ACC), vol. 145, pp. 947-952, 2011.

[37] S. Lee, J. Kim, J. Lee, and B. H. Cho, "State-of-charge and capacity estimation of lithium-ion battery using a new opencircuit voltage versus state-of-charge," Journal of Power Sources, vol. 185, no. 2, pp. 1367-1373, 2008.

[38] R. Xiong, F. Sun, Z. Chen, and H. He, "A data-driven multi-scale extended Kalman filtering based parameter and state estimation approach of lithium-ion polymer battery in electric vehicles," Applied Energy, vol. 113, pp. 463-476, 2014.

[39] J. Kim, S. Lee, and B. H. Cho, "Complementary cooperation algorithm based on DEKF combined with pattern recognition for SOC/capacity estimation and $\mathrm{SOH}$ prediction," IEEE Transactions on Power Electronics, vol. 27, no. 1, pp. 436-451, 2012.

[40] C. Hu, B. D. Youn, and J. Chung, "A multiscale framework with extended Kalman filter for lithium-ion battery SOC and capacity estimation," Applied Energy, vol. 92, pp. 694-704, 2012.

[41] L. Zheng, L. Zhang, J. Zhu, G. Wang, and J. Jiang, "Co-estimation of state-of-charge, capacity and resistance for lithiumion batteries based on a high-fidelity electrochemical model," Applied Energy, vol. 180, pp. 424-434, 2016.

[42] Z. Wei, K. J. Tseng, N. Wai, T. M. Lim, and M. Skyllas-Kazacos, "Adaptive estimation of state of charge and capacity with online identified battery model for vanadium redox flow battery," Journal of Power Sources, vol. 332, pp. 389-398, 2016.

[43] Z. Wei, J. Zhao, D. Ji, and K. J. Tseng, "A multi-timescale estimator for battery state of charge and capacity dual estimation based on an online identified model," Applied Energy, vol. 204, pp. 1264-1274, 2017.

[44] V. Duong, H. A. Bastawrous, K. Lim, K. W. See, P. Zhang, and S. X. Dou, "Online state of charge and model parameters estimation of the LiFePO4 battery in electric vehicles using multiple adaptive forgetting factors recursive least-squares," Journal of Power Sources, vol. 296, pp. 215-224, 2015.

[45] Y. Naderahmadian, M. A. Tinati, and S. Beheshti, "Generalized adaptive weighted recursive least squares dictionary learning," Signal Processing, vol. 118, pp. 89-96, 2016. 
[46] M. Badoni, A. Singh, and B. Singh, "Variable forgetting factor recursive least square control algorithm for DSTATCOM," IEEE Transactions on Power Delivery, vol. 30, no. 5, pp. 2353-2361, 2015.

[47] C. Chen and R. Xiong, "A lithium-ion battery-in-the-loop approach to test and validate multi-scale dual $\mathrm{H}$ infinity filters for state of charge and capacity estimation," IEEE Transactions on Power Electronics, pp. 1-11, 2017. 


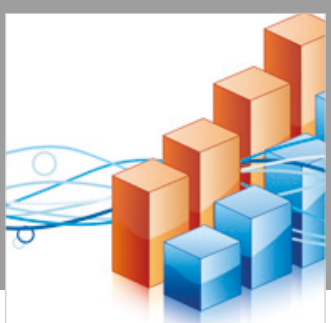

Advances in

Operations Research

\section{-n-m}
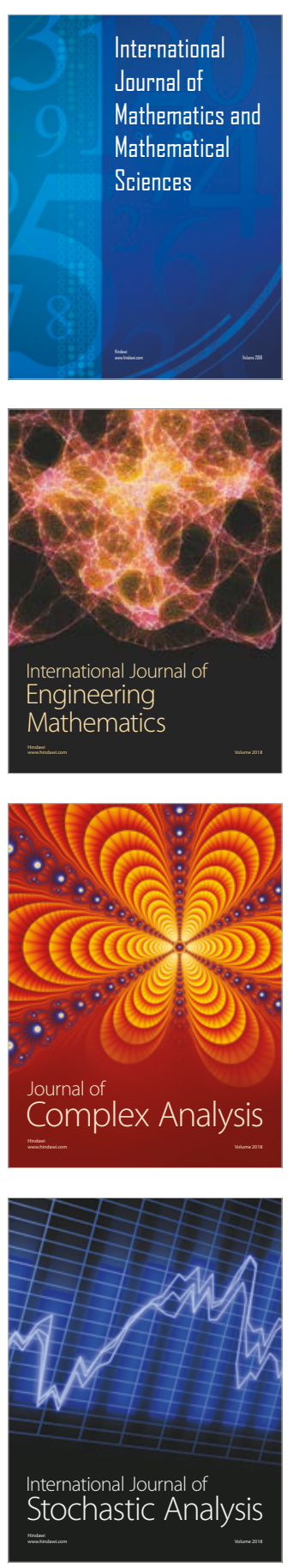
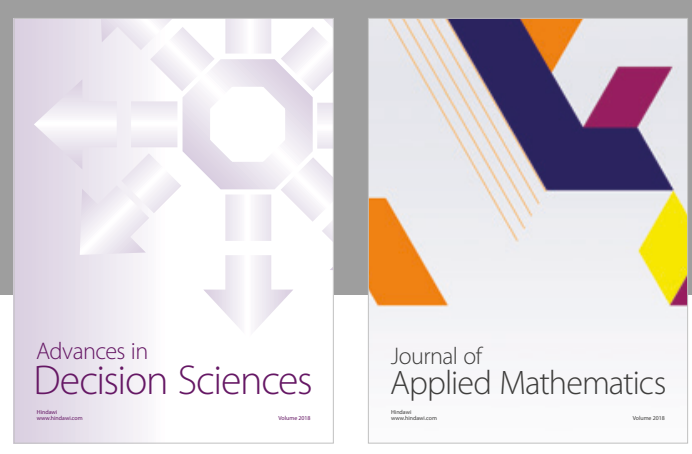

Journal of

Applied Mathematics
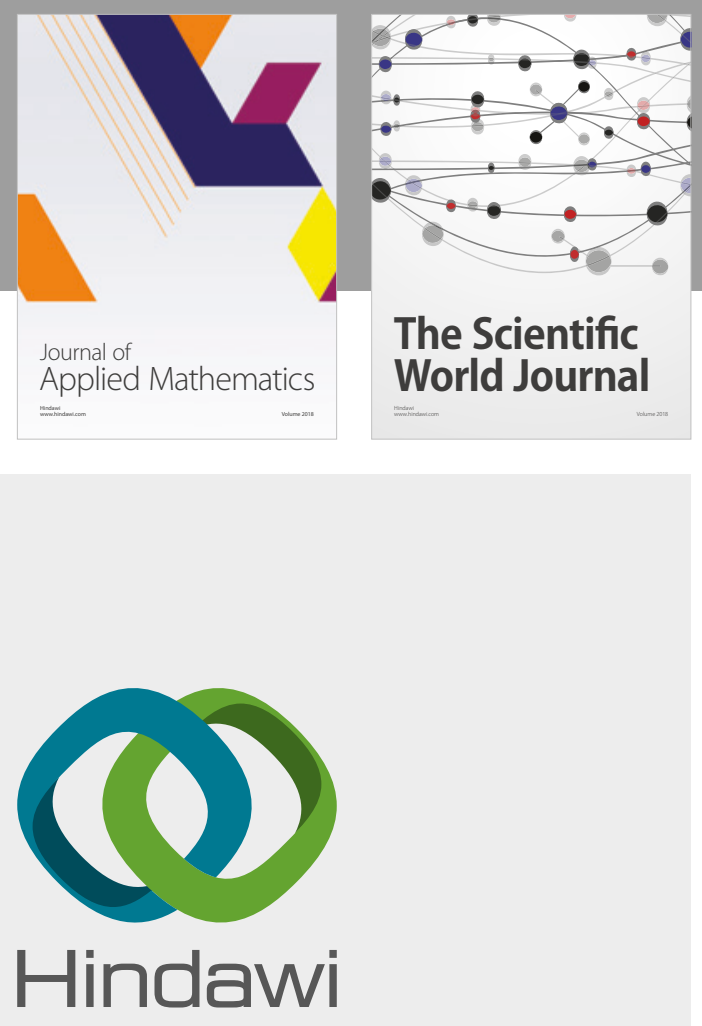

Submit your manuscripts at

www.hindawi.com

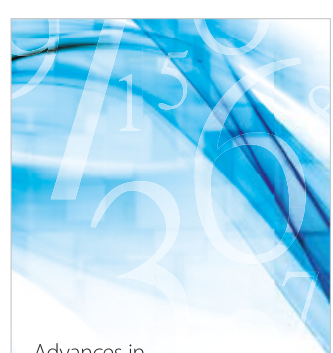

Advances in
Numerical Analysis
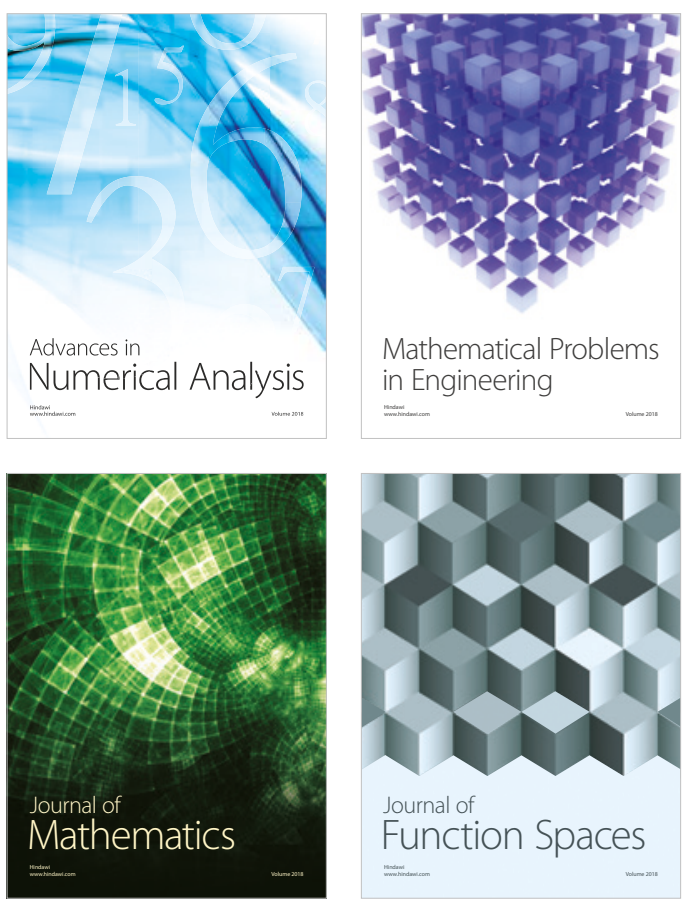

Mathematical Problems in Engineering

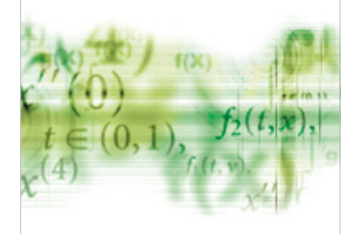

International Journal of

Differential Equations

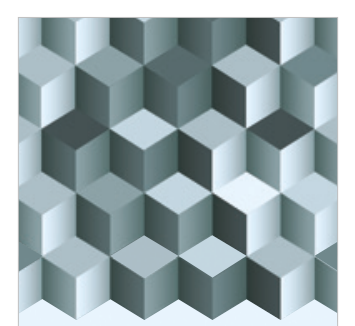

Journal of

Function Spaces

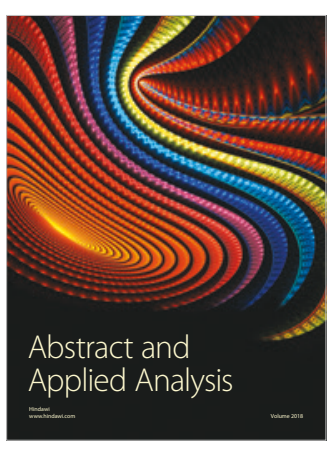

The Scientific

World Journal

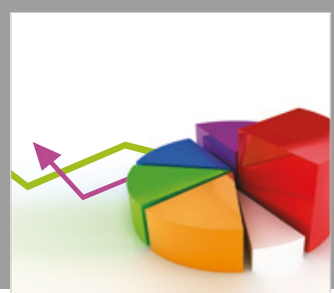

Journal of

Probability and Statistics
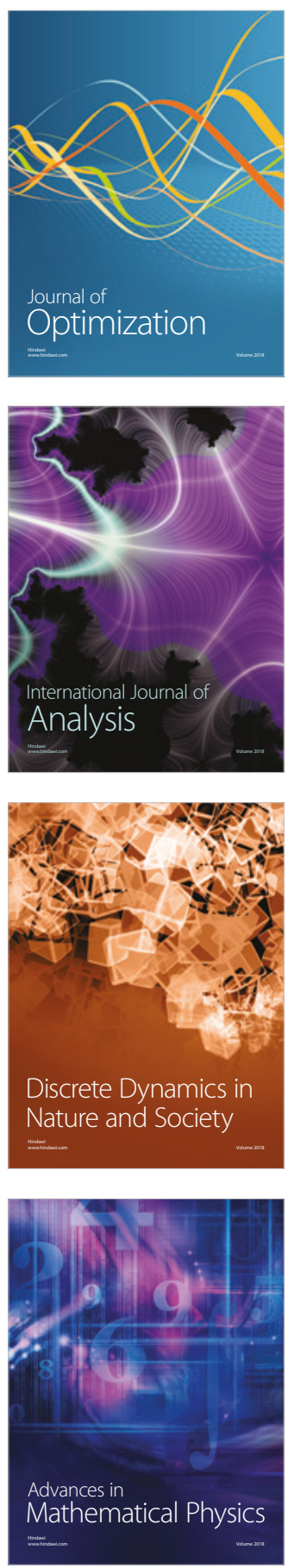